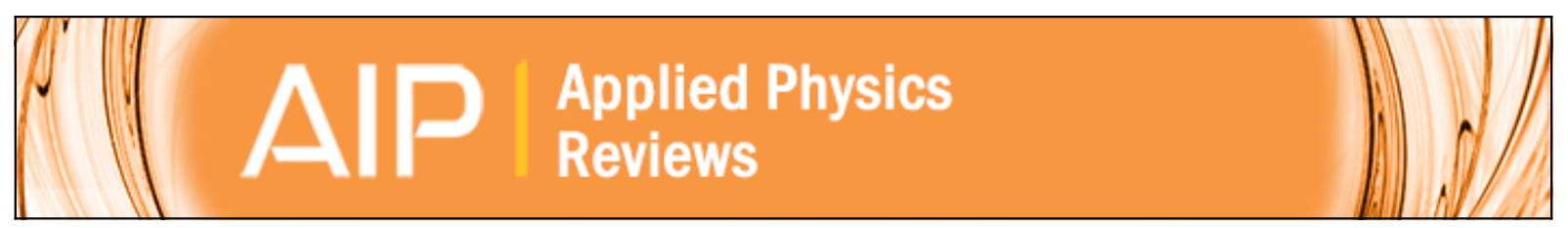

\title{
Multiferroic oxide thin films and heterostructures
}

Chengliang Lu, Weijin Hu, Yufeng Tian, and Tom Wu

Citation: Applied Physics Reviews 2, 021304 (2015); doi: 10.1063/1.4921545

View online: http://dx.doi.org/10.1063/1.4921545

View Table of Contents: http://scitation.aip.org/content/aip/journal/apr2/2/2?ver=pdfcov

Published by the AIP Publishing

\section{Articles you may be interested in}

Study of multiferroic properties in Bi5Fe0.5Co0.5Ti3O15 thin films

J. Appl. Phys. 111, 124116 (2012); 10.1063/1.4730892

Misfit strain induced magnetoelectric coupling in thin ferroic films

J. Appl. Phys. 105, 084108 (2009); 10.1063/1.3108483

Structural, magnetic, and ferroelectric properties of multiferroic Bi Fe O 3 film fabricated by chemical solution deposition

J. Appl. Phys. 101, 09M103 (2007); 10.1063/1.2711279

Multiferroic nanoparticulate $\mathrm{Bi} 3.15 \mathrm{Nd} 0.85 \mathrm{Ti} 3 \mathrm{O} 12$ - Co Fe $2 \mathrm{O} 4$ composite thin films prepared by a chemical solution deposition technique

Appl. Phys. Lett. 90, 152903 (2007); 10.1063/1.2709946

Sc modified multiferroic $\mathrm{Bi} F \mathrm{Fe} 3$ thin films prepared through a sol-gel process

Appl. Phys. Lett. 90, 022901 (2007); 10.1063/1.2430652

\section{A|P| $\begin{aligned} & \text { Journal of } \\ & \text { Applied Physics }\end{aligned}$}

Journal of Applied Physics is pleased to announce André Anders as its new Editor-in-Chief 


\title{
Multiferroic oxide thin films and heterostructures
}

\author{
Chengliang Lu, ${ }^{1, \mathrm{a})}$ Weijin $\mathrm{Hu}^{2}$ Yufeng Tian, ${ }^{3}$ and Tom $\mathrm{Wu}^{2, \mathrm{a})}$ \\ ${ }^{1}$ School of Physics and Wuhan National High Magnetic Field Center, Huazhong University of Science \\ and Technology, Wuhan 430074, China \\ ${ }^{2}$ Physical Sciences and Engineering Division, King Abdullah University of Science and Technology (KAUST), \\ Thuwal 23955-6900, Saudi Arabia \\ ${ }^{3}$ School of Physics, Shandong University, Jinan 250100, China
}

(Received 21 March 2015; accepted 1 April 2015; published online 26 May 2015)

\begin{abstract}
Multiferroic materials promise a tantalizing perspective of novel applications in next-generation electronic, memory, and energy harvesting technologies, and at the same time they also represent a grand scientific challenge on understanding complex solid state systems with strong correlations between multiple degrees of freedom. In this review, we highlight the opportunities and obstacles in growing multiferroic thin films with chemical and structural integrity and integrating them in functional devices. Besides the magnetoelectric effect, multiferroics exhibit excellent resistant switching and photovoltaic properties, and there are plenty opportunities for them to integrate with other ferromagnetic and superconducting materials. The challenges include, but not limited, defectrelated leakage in thin films, weak magnetism, and poor control on interface coupling. Although our focuses are Bi-based perovskites and rare earth manganites, the insights are also applicable to other multiferroic materials. We will also review some examples of multiferroic applications in spintronics, memory, and photovoltaic devices. (C) 2015 AIP Publishing LLC.
\end{abstract}

[http://dx.doi.org/10.1063/1.4921545]

\section{TABLE OF CONTENTS}

I. INTRODUCTION

A. General overview of multiferroic materials .

B. Synthesis of multiferroic thin films and strain engineering $\ldots \ldots \ldots \ldots \ldots \ldots \ldots$

C. Structure of the review................

II. MULTIFERROIC OXIDE THIN FILMS ........

A. Bi-containing multiferroic thin films .......

1. $\mathrm{BiFeO}_{3} \ldots \ldots \ldots \ldots \ldots \ldots \ldots \ldots \ldots$

2. $\mathrm{BiMnO}_{3}(\mathrm{BMO}) \ldots \ldots \ldots \ldots \ldots \ldots \ldots$

3. $\mathrm{BiCrO}_{3}(\mathrm{BCO}) \ldots \ldots \ldots \ldots \ldots \ldots \ldots$

4. Bi-based ordered double perovskites ....

B. Multiferroic rare-earth manganites ........

1. Antiferromagnetic $\mathrm{RMnO}_{3} \ldots \ldots \ldots \ldots$

2. Orthorhombic $\mathrm{RMnO}_{3}$ with spiral spin order (SSO) . . . . . . . . . . . . . . . . .

C. Other multiferroic thin films .............

D. Magnetism in multiferroic thin films.......

III. DEVICES BASED ON MULTIFERROIC THIN

FILMS. . . . . . . . . . . . . . . . . .

A. Resistive switching devices............

B. Ferroelectric/multiferroic tunnel junctions...

C. Multiferroic/ferromagnetic heterostructures .

\footnotetext{
a) Authors to whom correspondence should be addressed. Electronic addresses: cllu@mail.hust.edu.cn and Tao.Wu@kaust.edu.sa.
}

D. Multiferroic/superconducting heterostructures ................... 12

E. Photovoltaic effect in multiferroic thin films 13 IV. FINAL REMARKS AND OUTLOOKS ....... 14

\section{INTRODUCTION}

\section{A. General overview of multiferroic materials}

The terminology of multiferroics refers to materials where two or more ferroic orders such as ferroelectricity, (anti-)ferromagnetism, and ferroelasticity co-exist. More importantly, the strong coupling between multiple ferroic orders can produce additional functionalities, in particular, the magnetoelectric (ME) effect, which enables the control of electric polarization by a magnetic field and the control of magnetization by an electric field. Therefore, the enthusiastic endeavors on multiferroics have been motivated by not only the rich physics underlying the intricate spin-charge ordering in these materials but also their promising potential applications for future multifunctional computing and memory devices. $^{1-4}$

Research on the magnetoelectric effect can be traced back to the work by Dzyaloshinskii on $\mathrm{Cr}_{2} \mathrm{O}_{3}$ in 1960s. ${ }^{5}$ Within the next decade, there were some studies on the bulk composites of magnetostrictive ferrites and piezoelectric barium titanate. ${ }^{6}$ However, the research was halted for a number of years because the observed ME coupling was quite weak. Since 2003, the discoveries of spin-dependent multiferroicity and strong $\mathrm{ME}$ effect in $\mathrm{TbMnO}_{3}(\mathrm{TMO})^{7}$ and the 
coexistence of magnetism and excellent ferroelectricity in $\mathrm{BiFeO}_{3}(\mathrm{BFO})^{8}$ have boosted great interest in multiferroic materials. Up to date, over ten different multiferroic material families have been extensively investigated, including the rare-earth manganates and the well-known BFO. Broadly speaking, these materials can be divided into single phase multiferroics with widely separated ferroelectric and magnetic ordering temperatures (type I multiferroic), singe phase multiferroics having a magnetic transition with concurrent ferroelectric ordering (type II multiferroic), and composite multiferroics formed by distinct but coupled ferromagnetic (FM) and ferroelectric (FE) components. ${ }^{9}$ However, most of the single-phase multiferroics reported so far are antiferromagnets without spontaneous magnetization, and generally their Curie or Néel temperatures are far below the room temperature, diminishing their chance of practical applications.

The scarcity of multiferroic materials stems from the fact that magnetism requires unpaired $d^{\mathrm{n}}$ cations, while ferroelectricity exists almost exclusively in $d^{0}$ compounds. ${ }^{10}$ This intrinsic constraint has invited lots of efforts on searching new multiferroics as well as on developing relevant theoretical models. This research thrust has witnessed many synergetic collaborations between experimentalist and theorists, and in many cases the modern advancements of firstprinciples calculations help to shed light on the physics of multiferroics. ${ }^{11}$

\section{B. Synthesis of multiferroic thin films and strain engineering}

It is not an exaggeration to say, that the research on multiferroic thin films was enabled by the developments of stateof-the-art thin film deposition tools. With careful tuning of synthesis conditions, the multiferroic properties of thin films could be enhanced compared to those of the bulk counterparts. For example, BFO was extensively investigated in 1960 s, but its spontaneous polarization was revealed to be just few micro-Coulombs per square centimeter, owing to the leakage problems associated with the low crystal quality. ${ }^{12-14}$ This issue was eventually solved by high-quality thin film growth using pulsed laser deposition (PLD) in 2003, which is one of the milestones in the field of multiferroics. ${ }^{8}$

Successful thin film growth is the foundation of almost all device applications, but it introduces extrinsic complications such as substrate strain, surface states, impurity phases, structural defects, and so on. For example, excessive leakage current may compromise the measurement of ferroelectricity; surfaces and interfaces may introduce spin canting, which compromises magnetism. Thus, it is important to secure strategies of retaining or even improving the multiferroic properties in thin films.

Various state-of-art physical deposition techniques, such as PLD, molecular beam epitaxy (MBE), and sputtering, and chemical synthesis routes, such as sol gel spin coating and metal-organic chemical vapor deposition (MOCVD), have been utilized to prepare multiferroic thin films. PLD is a physical vapor deposition method that is widely used in the research community, particularly for investigating new materials and novel heterostructures. An intrinsic advantage of PLD technique is that the growth process takes place far from equilibrium and hence it is good at preserving complex stoichiometry, which is especially important for the growth of multi-element compounds. Moreover, being equipped with in-site reflection high-energy electron diffraction (RHEED), PLD enables the precise control of film thickness on the unit cell level, ${ }^{15}$ which is critical for the synthesis of high-quality epitaxial thin films. In contrast to PLD, MBE involves the generation of thermal atomic beams with very low energy $(\sim 1 \mathrm{eV})$, which is quite suitable to prepare extremely pure and defect free thin films of multiferroics and other complex oxides. ${ }^{16}$ Although PLD and MBE are capable to produce high quality films, such processes are challenging to scale up to large-area wafers. Sputtering, on the other hand, is a widely used deposition techniques for largescale production, including magnetron sputtering, ion-beam sputtering, and off-axis sputtering. ${ }^{17}$ Precise control of reactive gas, especially the $\mathrm{O}_{2}$ or $\mathrm{Ar} / \mathrm{O}_{2}$ mixtures, is crucial for synthesizing high-quality oxide thin films with desired oxygen stoichiometry. Different from the vapor-based methods, sol gel spin coating is a convenient and inexpensive chemical process to fabricate thin films with large areas and low costs. However, the obtained films are usually in the polycrystal or textured structures, and it is often hard to tune the sample thickness and morphology. Finally, MOCVD has been routinely used in the electronics industry, which offers promising potential for the deposition of multiferroic films due to several advantages, such as excellent film uniformity over large areas, relatively high deposition rates, and compatibility with modern integrated circuit technologies, ${ }^{18}$ but there are limited metal-organic sources for growing complex oxides. More information on the growth of ferroelectric and multiferroic oxide thin films can be found in a few recent reviews. ${ }^{18,19}$

Selecting suitable substrates is often the first step for the thin film synthesis. The crystal structure and lattice parameters of several widely studied multiferroic materials and some typical substrates are listed in Table I. On the one hand, good lattice match between film and substrate is a prerequisite for growing smooth films with controlled low-density structural defects. On the other hand, strain (either tensile or compressive) engineering is an effective tool to induce lattice distortion and to optimize physical properties. ${ }^{20}$

Strain engineering, in fact, is a general strategy often employed in semiconductor industry to increase the carrier mobility in the transistor channel and to enhance device performance. For example, capping layers of CVD silicon nitride are used to induce either compressive strain or tensile strain to improve the channel transport. Other methods like growing epitaxial silicon on silicon-germanium underlayer are also adapted to modulate the channel strain. It has been reported for ferroelectric oxides that strain engineering can lead to sizable increases of spontaneous polarization and Curie temperature. ${ }^{21}$ Similarly, the properties of epitaxial multiferroic thin films can be engineered by substrate-induced strain. Strain engineering has been extensively exploited for BFO thin films. One of the most notable examples is the strain induced tetragonal BFO phase which possesses giant $c / a$ ratio $(>1.25)$ and 
TABLE I. Lattice parameters and crystal structure of several typical multiferroics and substrates. ${ }^{29-36}$

\begin{tabular}{lcc}
\hline \hline & Crystal structure & Lattice parameters \\
\hline $\mathrm{Compound}$ & & $a=3.965 \AA$ \\
$\mathrm{BiFeO}_{3}$ & Rhombohedral & $a=b=3.935 \AA, c=3.989 \AA$ \\
$\mathrm{BiMnO}_{3}$ & Triclinic & $a=b=3.90 \AA, c=3.87 \AA$ \\
$\mathrm{BiCrO}_{3}$ & Triclinic & $a=6.13 \AA, c=11.4 \AA$ \\
$\mathrm{YMnO}_{3}$ & Hexagonal & $a=5.2418 \AA, b=5.8029 \AA, c=7.3643 \AA$ \\
& Orthorhombic & $a=5.2931 \AA, b=5.8384 \AA, c=7.4025 \AA$ \\
$\mathrm{TbMnO}_{3}$ & Orthorhombic & $a=5.2785 \AA, b=5.8337 \AA, c=7.3778 \AA$ \\
$\mathrm{DyMnO}_{3}$ & Orthorhombic & $a=5.1984 \AA, b=5.7845 \AA, c=7.2994 \AA$ \\
$\mathrm{LuMnO}_{3}$ & Orthorhombic & \\
$\mathrm{Substrate}$ & & $a=3.905 \AA$ \\
$\mathrm{SrTiO}_{3}$ & & $a=3.792 \AA$ \\
$\mathrm{LaAlO}_{3}$ & Cubic & $a=3.868 \AA$ \\
$\left(\mathrm{LaAlO}_{3}\right)_{0.3}\left(\mathrm{LaSr}_{2} \mathrm{TaO}_{6}\right)_{0.7}$ & \\
$\mathrm{LaSrAlO}_{4}$ & Pseudocubic & $a=3.754 \AA, c=11.263 \AA$ \\
$\mathrm{LaSrGa}_{4}$ & Tetragonal & $a=3.84 \AA, c=12.676 \AA$ \\
$\mathrm{NdScO}_{3}$ & Tetragonal & $a=5.777 \AA, b=5.577 \AA, c=8.005 \AA$ \\
$\mathrm{YAlO}_{3}$ & Tetragonal & $a=5.18 \AA, b=5.33 \AA, c=7.37 \AA$ \\
$\mathrm{NdGaO}_{3}$ & Orthorhombic & $a=5.417 \AA, b=5.499 \AA, c=7.717 \AA$ \\
$\mathrm{DyScO}_{3}$ & Orthorhombic & $a=5.54 \AA, b=5.71 \AA, c=7.89 \AA$ \\
\hline \hline
\end{tabular}

spontaneous polarization up to $150 \mu \mathrm{C} / \mathrm{cm}^{2,22-25}$ Since the tetragonal BFO was identified in $2009,{ }^{22}$ significant progresses have been achieved on the related research. ${ }^{26}$ One impressive feature of this tetragonal phase BFO is the reversible control of its crystalline structure between rhombohedra and tetragonal using an electric field, which provides the possibility of making nanoscale shape-memory devices. ${ }^{27}$ While tetragonal BFO has been intensively addressed, orthorhombic BFO has also attracted lots of interest due to its interesting physical properties. ${ }^{28}$

Strain can significantly alter the phase diagrams of oxides with strong correlations between multiple degrees of freedom. For example, in systems with strong spin-phonon coupling, if the lowest-frequency polar phonon is softer for ferromagnetic ordering than for antiferromagnetic (AFM) ordering, then strain may induce a polar instability and trigger transition from the antiferromagnetic-paraelectric state to the ferromagneticferroelectric state. This strain-induced multiferroic state was proposed for $\mathrm{SrMnO}_{3}$ based on the results of first-principle calculations, encouraging experimental efforts to explore thin films of materials with strong spin-phonon coupling. ${ }^{37}$

\section{Structure of the review}

In Sec. I, we have given a general overview of multiferroics and briefly reviewed the aspects of thin film growth and strain engineering. The main aim of this review is to discuss the most recent works on multiferroic thin films and devices, with a focus on the perspective of novel applications. In spite of recent progresses, growing thin films with retained or improved multiferroic functionalities remains as a challenge and presents opportunities of breakthroughs at the same time. ${ }^{38-40}$ We should note that this review is constraint to the research related to multiferroic thin films. Significant progresses have been made on the front of multiferroic bulk samples in the form of single crystals, ceramics, and multi-component thick layers. ${ }^{41}$ There are also interesting works on non-oxide multiferroics such as $\mathrm{CuCl}_{2}$. ${ }^{42}$ Our aim here is not to exhaust the fascinating physics in the broad field of multiferroics, but to give the readers a glimpse of recent discoveries on multiferroic oxide thin films, and to offer a perspective of possible future research directions.

In Sec. II, we will review the research progresses made on a few prototypical multiferroic oxides, particularly $\mathrm{Bi}$ containing compounds and rare-earth manganites. We should note here that BFO is probably the most investigated multiferroic material, and there have been several comprehensive reviews in the literature. ${ }^{39,43-45}$ This review will not duplicate the existing ones, and we will give more attention to other multiferroic oxides. It is a consensus in the community that more efforts are needed to improve the materials synthesis and to elucidate the physical properties of many multiferroic compounds. This section also includes a brief discussion on the magnetism of multiferroic oxide thin films. Although the conventional antiferromagnetic order in some wellstudied multiferroics does not give rise to sizable magnetism in bulk samples, a few works reported some weak magnetic signals in thin films. Such a size effect is particularly important for nanoscale and interface-based devices, and the underlying mechanisms will be discussed.

In Sec. III, we will review several device prototypes involving multiferroic thin films. The most straightforward one in terms of device fabrication and characterization is the resistive switching devices, where the memory functionality hinges on the toggles between high and low resistance states. A related device concept, but involving the ferroelectricity in ultrathin multiferroic thin films, is the ferroelectric tunneling junctions (FTJs). In the third device application, researchers take advantage of the interfacial coupling between multiple ferroic orders to realize new ME controls in multiferroic/ ferromagnetic heterostructures. Although much less investigated, multiferroic/superconducting heterostructures 
constitute one particular type of fascinating multiferroic thin film device. Last but not least, we will briefly discuss the photovoltaic (PV) devices involving multiferroic thin films, and open questions remain regarding the transport physics of such oxide films with domain walls under the light stimulation. Although time is still too early to unambiguously predict the killer applications of multiferroic materials, their fascinating properties continue inviting research efforts from both theoretical and experimental communities.

This review ends with a summary and outlooks in Sec. IV.

\section{MULTIFERROIC OXIDE THIN FILMS}

\section{A. Bi-containing multiferroic thin films}

\section{1. $\mathrm{BiFeO}_{3}$}

In the arena of multiferroic research, one of the most successful stories is $\mathrm{BiFeO}_{3}$, which exhibits room temperature ferroelectric and magnetic orders $\left(T_{\mathrm{C}}=830^{\circ} \mathrm{C}\right.$ and $T_{\mathrm{N}}=370^{\circ} \mathrm{C}$ ). The ordering of highly polarizable $\mathrm{Bi}$ lone pairs drives the emergence of ferroelectric polarization, while the super-exchange between neighboring $\mathrm{Fe}$ and $\mathrm{O}$ ions leads to the G-type antiferromagnetic order. Bulk BFO has a rhombohedral lattice structure (space group: $R 3 \mathrm{c}$ ) with lattice parameters of $a_{\mathrm{hex}}=5.58 \AA$ and $c_{\mathrm{hex}}=13.90 \AA$. It perovskite-type unit cell has a lattice parameter, $a=3.965 \AA^{8,29,44}$

$\mathrm{BFO}$ thin films present complex and fascinating domain physics. The ferroelectric domain structure of BFO films can be characterized by analyzing the in-plane (IP) and out-ofplane (OOP) piezoelectric force microscopy (PFM) images. Three types of domain walls can be identified, e.g., $71^{\circ}, 109^{\circ}$, and $180^{\circ}$, as illustrated in Figure 1(a). ${ }^{43}$ At present, people can successfully control the domain configurations during or after the growth of BFO films (Figures 1(b)-1(d)). ${ }^{46-50}$ Some interesting domain-related physics have been revealed in BFO films with carefully controlled ferroelectric domains, such as the high conductivity in domain wall ${ }^{51}$ and abovebandgap photovoltage. ${ }^{52,53}$

\section{2. $\mathrm{BiMnO}_{3}(\mathrm{BMO})$}

$\mathrm{BMO}$ is a unique multiferroic material, possessing ferromagnetic $\left(T_{\mathrm{FM}}=105 \mathrm{~K}\right)$ and ferroelectric $\left(T_{\mathrm{FE}}=770 \mathrm{~K}\right)$ ground state with a spontaneous polarization $\left(P \sim 16 \mu \mathrm{C} / \mathrm{cm}^{2}\right)$ and magnetization $\left(M \sim 3.6 \mu_{\mathrm{B}} / \mathrm{Mn}\right) .^{30,54-58}$ Similar to $\mathrm{BFO}$, the ferroelectric state in $\mathrm{BMO}$ is caused by the stereochemical activity of $\mathrm{Bi} 6 \mathrm{~s}^{2}$ lone pairs, while the ferromagnetism originates from the ferromagnetic super-exchange interaction between $\mathrm{Mn}^{3+}$. High pressure is required for the synthesis of bulk BMO single crystals, but this difficulty can be overcome by epitaxial stabilization in thin films. Epitaxial BMO thin films have been successfully grown using PLD by a few groups, and the growth window appears to be very narrow. ${ }^{31,59-64}$ The magnetic property of BMO film is very sensitive to the growth parameters, which can be associated with the Bi vacancy and the substrate strain effect. ${ }^{31,61,62}$ To avoid these shortcomings, Gajek et al. partially replaced $\mathrm{Bi}^{3+}$ with isovalent $\mathrm{La}^{3+}$ while retaining the good FE-FM property. They found a broader growth window, and the magnetism is more stable than the case of pure BMO thin films. ${ }^{65}$

In terms of devices, a four-state resistive system was realized in a $\mathrm{La}_{0.1} \mathrm{Bi}_{0.9} \mathrm{MnO}_{3}(\mathrm{LBMO}) / \mathrm{La}_{2 / 3} \mathrm{Sr}_{1 / 3} \mathrm{MnO}_{3}$ heterostructure (Figure 2). ${ }^{66}$ The junction transport depends on not only the relative magnetic orientations like in the conventional magnetic tunneling junctions but also the polarization direction of the thin LBMO layer. It represents a significant breakthrough in the quest for the next-generation memory devices.

\section{3. $\mathrm{BiCrO}_{3}(\mathrm{BCO})$}

$\mathrm{BCO}$ is another typical perovskite multiferroic material with large $\mathrm{A}$-site $\mathrm{Bi}^{3+}$, in which antiferroelectricity and (a)
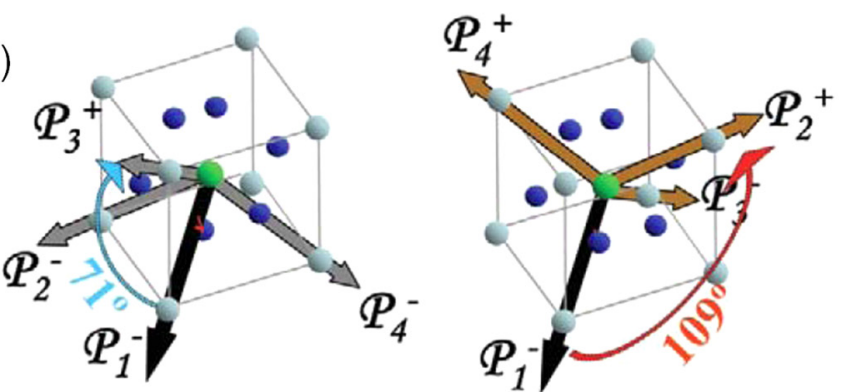

(b)

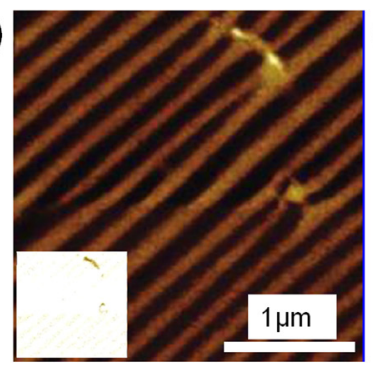

(c)

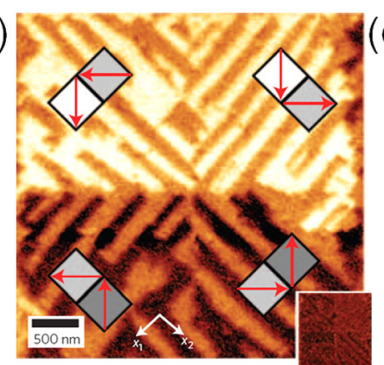

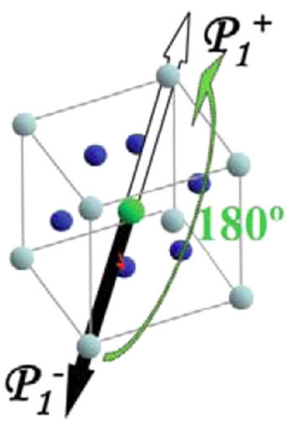

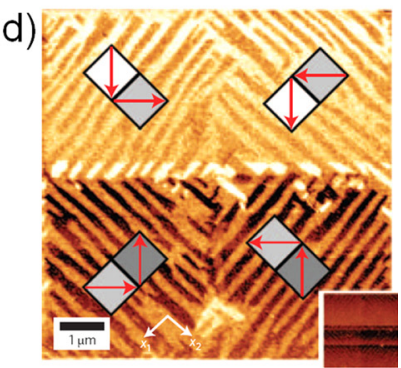

FIG. 1. (a) Three possible mechanisms for switching the polarization in $\mathrm{BiFeO}_{3}$. Reprinted with permission Chu et al., Mater. Today 10, 16 (2007). Copyright 2007 Elsevier. (b) In-plane PFM image reveals clear stripe domains which are formed during the film deposition. The inset shows the out-of-plane PFM image of BFO thin film, in which no evident contrast can be observed. Reprinted with permission from Chu et al., Nano Lett. 9, 1726 (2009). Copyright 2009 American Chemical Society. (c) and (d) In-plane PFM images of artificial domain patterns with different configurations for $\mathrm{BiFeO}_{3}$ thin films grown on $\mathrm{SrTiO}_{3}$ (001) substrates with $\mathrm{La}_{0.7} \mathrm{Sr}_{0.3} \mathrm{MnO}_{3}$ buffer layers. The insets show the corresponding out-of-plane PFM images. Reprinted with permission from Balke et al., Nat. Nanotechnol. 4, 868 (2009). Copyright 2009 Nature Publishing Group. 
(a)
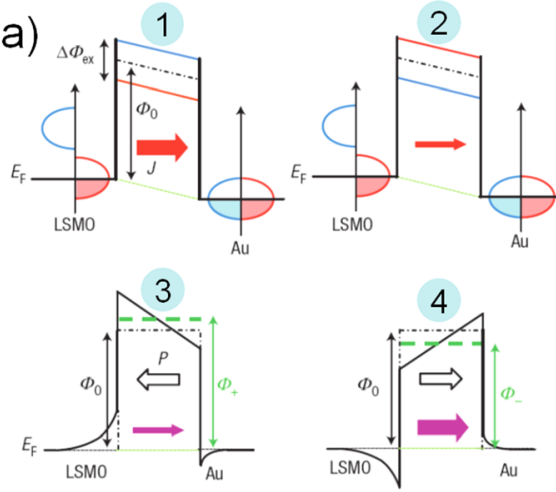

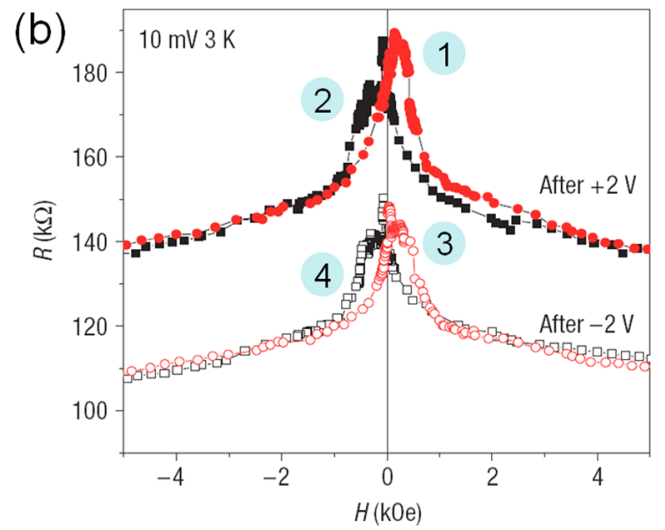

FIG. 2. (a) Schematic drawing of the tunnel barrier potential profiles and tunnel currents in the LSMO/LBMO/Au heterostructure. Upper figures indicate spindependent tunneling for parallel (left) or antiparallel (right) configuration of the LBMO and LSMO magnetization. Bottom figures indicate polarization dependent tunneling for electric field parallel (left) or antiparallel (right) to the polarization; (b) tunnel magnetoresistance of the junction measured at $3 \mathrm{~K}$ at $10 \mathrm{mV}$ after applying a positive (2 V) or negative (-2 V) voltage across the junction. Reprinted with permission from Gajek et al., Nat. Mater. 6, 296 (2007). Copyright 2007 Nature Publishing Group.

weak parasitic ferromagnetism were evidenced. ${ }^{32,67}$ Unlike BFO, only a few studies on BCO thin films have been reported. Hill first theoretically predicted in BCO a G-type antiferromagnetic ground state with antiferroelectric structure distortion. ${ }^{68,69}$ Murakami et al. ${ }^{32}$ reported piezoelectric response and tunable dielectric constant in BCO epitaxial thin film grown on $\mathrm{LaAlO}_{3}$ (LAO) substrate, while weak ferromagnetism arises at low temperatures. Similar weak ferromagnetism was revealed by Kim et $a l .^{67}$ in BCO thin films grown on $\mathrm{SrTiO}_{3}$ (STO) (001) substrates, while double hysteresis loops were observed in both $\varepsilon(E)$ and $P(E)$, indicating the antiferroelectricity within the film, as shown in Figure 3. There remain open questions regarding the antiferroelectricity and weak ferromagnetism in BCO thin films, while multiphase coexistence may help to understand these features. ${ }^{70}$

\section{Bi-based ordered double perovskites}

For the Bi-based transition metal perovskites $\mathrm{BiMO}_{3}$ $(\mathrm{M}=\mathrm{Fe}, \mathrm{Mn}, \mathrm{Cr}, \ldots)$, ordered double perovskites (such as $\mathrm{BiFe}_{0.5} \mathrm{Cr}_{0.5} \mathrm{O}_{3}$ and $\mathrm{BiFe}_{0.5} \mathrm{Mn}_{0.5} \mathrm{O}_{3}$ ) were theoretically predicted to be promising multiferroic candidates, in which large spontaneous polarization and ferromagnetism could be realized simultaneously. ${ }^{69,71}$ Indeed, very large polarization $\left(P \sim 60 \mu \mathrm{C} / \mathrm{cm}^{2}\right)$ was experimentally evidenced in
$\mathrm{BiFe}_{0.5} \mathrm{Cr}_{0.5} \mathrm{O}_{3}$ thin film, but the magnetism is weak (Figures 4(a) and 4(b)). ${ }^{72}$

A notably large magnetization of $\sim 90 \mathrm{emu} / \mathrm{cc}$ was reported in $\mathrm{BiFe}_{0.5} \mathrm{Mn}_{0.5} \mathrm{O}_{3}$ thin films (Figure $\left.4(\mathrm{c})\right)^{73}$ In a different approach, Nechache et al. fabricated $\mathrm{BFO} / \mathrm{BCO}$ heterostructures using PLD and revealed significant enhancement of magnetism. ${ }^{74}$

\section{B. Multiferroic rare-earth manganites}

An intriguing class of compounds is rare-earth (R) manganites that show either a hexagonal structure when the $\mathrm{R}$ ionic radius is small $(P 63 \mathrm{~cm}$, from $\mathrm{Y}, \mathrm{Sc}$, and Ho to $\mathrm{Lu})$ or an orthorhombic structure when the ions are large (Pnma, from Dy to La). The crossover is around Y/Ho (Figure 5). ${ }^{38}$ Recent studies demonstrated that the hexagonal manganites with small $\mathrm{R}$ ionic radius can be transformed into the orthorhombic phase through soft chemical synthesis, thin film growth, or high pressure treatment. Intriguing physical properties were revealed in these newly synthesized orthorhombic phases. ${ }^{33,75,76}$ Reversely, the orthorhombic phase can also be transformed to the hexagonal one through exploiting the epitaxial strain, and the associated physical properties can be modified correspondingly. ${ }^{34,77}$
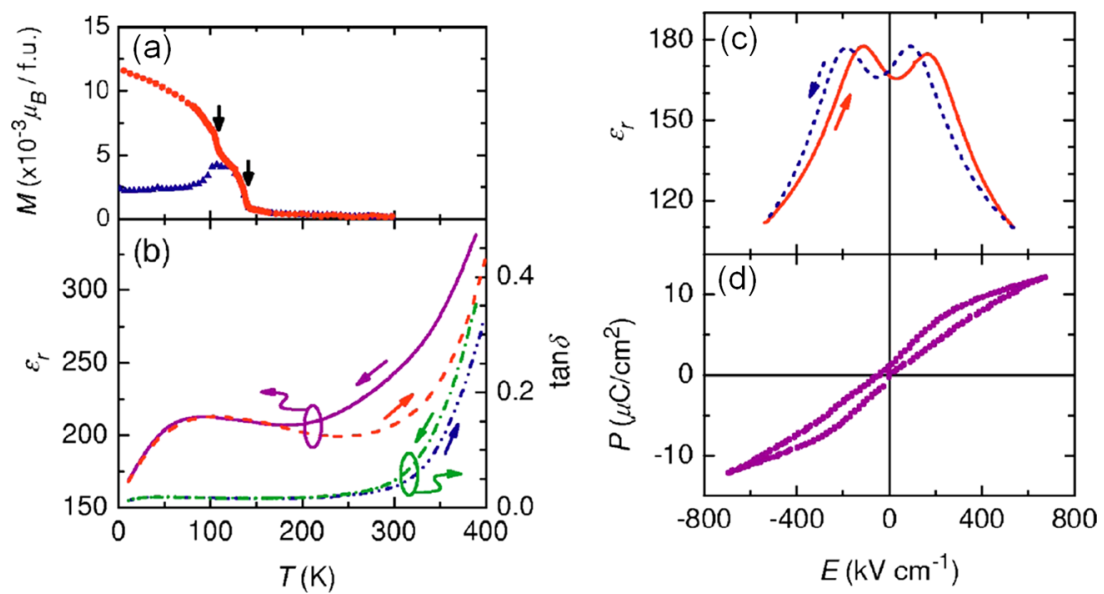

FIG. 3. (a) Temperature dependent magnetization of BCO film on STO substrate with a $\mathrm{SrRuO}_{3}$ bottom layer measured at $2 \mathrm{kOe}$ under field cooling (red circles) and zero field cooling (blue triangles) conditions. (b) Dielectric constant and loss tangent data as a function of temperature measured during cooling or warming processes. Dielectric constant (c) and polarization (d) as a function of electric field measured at a frequency of 10 $\mathrm{kHz}$. Reprinted with permission from Appl. Phys. Lett. 89, 162904 (2006). Copyright 2006 AIP Publishing LLC. 

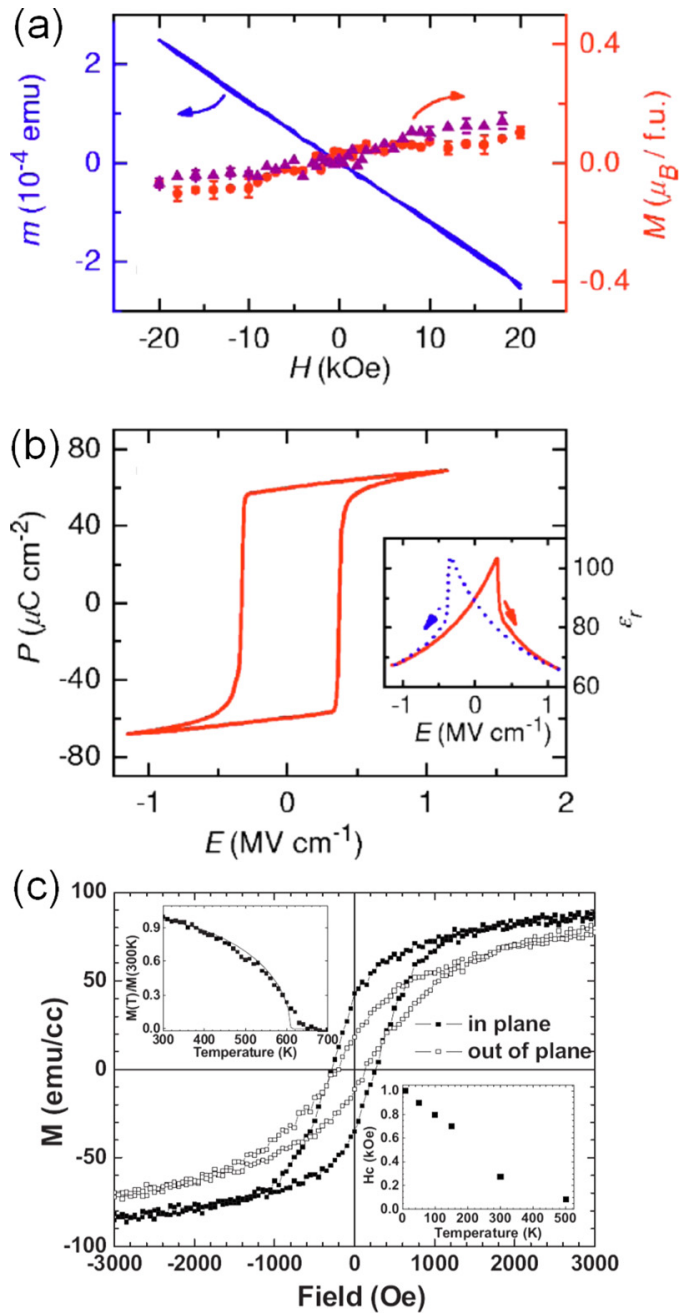

FIG. 4. (a) Magnetic field dependent magnetization for the $\mathrm{BiFe}_{0.5} \mathrm{Cr}_{0.5} \mathrm{O}_{3}$ film grown on $\mathrm{SrTiO}_{3}(001)$ substrate with a $\mathrm{SrRuO}_{3}$ bottom layer. The blue line is the original data, and the red circles (increasing field) and purple triangles (decreasing field) are the data after subtracting the diamagnetism from the substrate. (b) Ferroelectric hysteresis loop measured at $T=77.3 \mathrm{~K}$ with a frequency of $1 \mathrm{kHz}$. The inset shows the electric field dependent dielectric constant with a measuring frequency of $10 \mathrm{kHz}$ at $T=77.3 \mathrm{~K}$. Reprinted with permission from Appl. Phys. Lett. 91, 042906 (2007). Copyright 2007 AIP Publishing LLC. (c) Room temperature magnetic hysteresis loop for a $\mathrm{BiFe}_{0.5} \mathrm{Mn}_{0.5} \mathrm{O}_{3}$ film grown on $\mathrm{SrTiO}_{3}$ (001) substrate. Reprinted with permission from Appl. Phys. Lett. 98, 012509 (2011). Copyright 2011 AIP Publishing LLC.

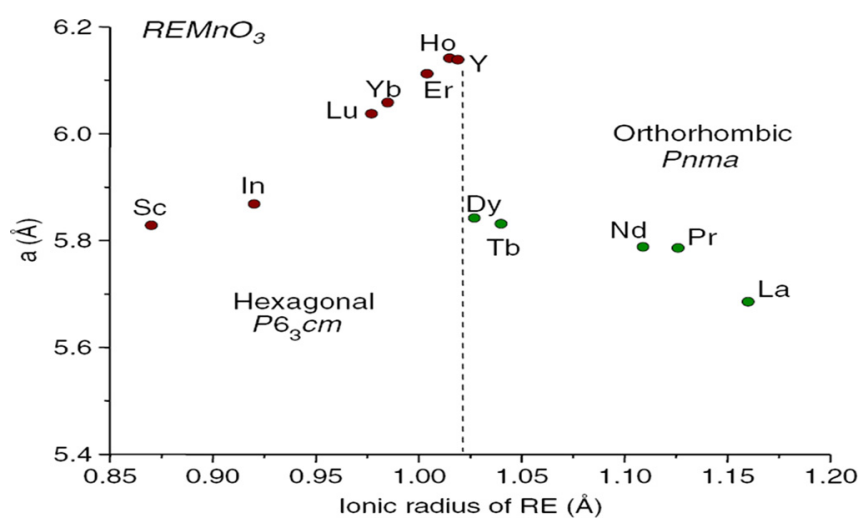

FIG. 5. Structural evolution of $\mathrm{RMnO}_{3}$ as a function of the radius of A-site rare earth ions. Adapted with permission from Prellier et al., J. Phys.: Condens. Matter 17, R803 (2005). Copyright 2005 Institute of Physics.

\section{Antiferromagnetic $\mathrm{RMnO}_{3}$}

The most studied hexagonal $\mathrm{RMnO}_{3}$ is $\mathrm{YMnO}_{3}$ (YMO), in which layers of $\mathrm{MnO}_{5}$ trigonal bipyramids (different from the $\mathrm{MnO}_{6}$ octahedron in the perovskites) and layers of $\mathrm{Y}^{3+}$ alternatively packed along the $c$ axis. The lattice parameters of YMO are $a=6.13 \AA$ and $c=11.4 \AA^{78}$ One obvious advantage of YMO is that it does not contain volatile elements such as $\mathrm{Bi}$ and $\mathrm{Pb}$. The spin configuration of hexagonal YMO is frustrated as a result of the geometric constraint. In the layer of $\mathrm{MnO}_{5}$ bipyramids ( $a b$ plane), the spins of $\mathrm{Mn}^{3+}$ are mediated by strong in-plane antiferromagnetic superexchange interaction and restricted within the plane. Along the $c$ axis, the inter-plane exchange between Mn spins is about two orders of magnitude lower than that within the $a b$ plane, giving rise to a strong anisotropy and an easy $a b$ plane. The long range antiferromagnetic order of Mn spins arises at $T_{\mathrm{N}} \sim 70 \mathrm{~K}$ with a $120^{\circ}$ configuration, which is typically observed in triangular antiferromagnets. Bulk single crystals of YMO possess a remnant polarization of $5.5 \mu \mathrm{C} /$ $\mathrm{cm}^{2}$. In early works in the 1960s, the ferroelectricity $\left(T_{\mathrm{C}} \sim 950 \mathrm{~K}\right)$ in hexagonal YMO was ascribed to an offcenter distortion of $\mathrm{Mn}^{3+}$ towards one of the apical oxygen ions. ${ }^{79,80}$ However, recent detailed single crystal $\mathrm{x}$-ray diffraction experiments revealed that the Mn ions remain very close to the centre of the oxygen bipyramids, and the ferroelectric phase should be ascribed to the buckling of the layered $\mathrm{MnO}_{5}$ bipyramids accompanied by the displacements of the $\mathrm{Y}$ ions. ${ }^{78}$ The mechanism of the ferroelectricity in hexagonal $\mathrm{YMO}$ is associated with the strong hybridization between $\mathrm{Y} 4 d$ and $\mathrm{O} 2 p$ states. $^{81}$

Since both ferroic orders are associated with the crystal structure, the ME coupling should be strong in YMO. Indeed, coupled magnetic and ferroelectric domains were directly mapped using the optical second harmonic generation (SHG) technique, as shown in Figure $6 .^{82,83}$ Recently, Choi et al. reported very interesting interlocked ferroelectric and structural antiphase domain walls in hexagonal YMO single crystals using transmission electron microscopy (TEM) and conductive atomic force microscopy. ${ }^{84}$ At the isostructural transition in hexagonal manganites, giant

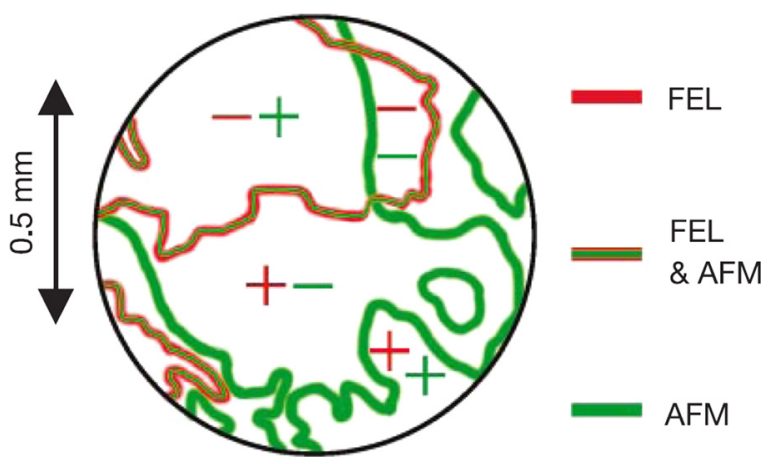

FIG. 6. Coupled magnetic and ferroelectric domain structures observed in $\mathrm{YMnO}_{3} . \mathrm{YMnO}_{3}$ has four types of $180^{\circ}$ domains denoted by $(+P,+l),(+P$, $-l),(-P,-l)$, and $(-P,+l)$, respectively, where $\pm \mathrm{P}$ and $\pm l$ are the independent components of the ferroelectric and antiferromagnetic order parameters. Reprinted with permission from Fiebig et al., Nature 419, 818 (2002). Copyright 2002 Nature Publishing Group. 
magneto-elastic coupling was observed and the atomic displacement is nearly $0.1 \AA^{85}$

YMO thin films have been grown on various substrates including $\mathrm{SrTiO}_{3}, \mathrm{MgO}, \mathrm{ZnO}, \mathrm{GaN}$, and $\mathrm{Si}$, in which the physical properties are qualitatively similar to the bulk counterpart. ${ }^{86-92}$ In ultrathin YMO films grown on yttriastabilized zirconia (111) substrates, strain can induce in-plane trigonality and four stable polarization states. ${ }^{93}$ The crystal structure of YMO can be dramatically changed to orthorhombic when it is grown on $\mathrm{YAlO}_{3}(010)$ or $\mathrm{SrTiO}_{3}$ substrate. In the orthorhombic-YMO (o-YMO), the superexchange interaction mediated long-range E-type antiferromagnetic order can directly generate ferroelectricity $\left(P \sim 0.8 \mu \mathrm{C} / \mathrm{cm}^{2}\right)$ due to the effect of exchange striction. ${ }^{76}$

In another E-type antiferromagnetic ferroelectric material $\mathrm{TmMnO}_{3}$, the spontaneous polarization was reported to be $0.45 \mu \mathrm{C} / \mathrm{cm}^{2} .{ }^{94}$ Hexagonal $\mathrm{HoMnO}_{3}$ shows a higher polarization of $5.6 \mu \mathrm{C} / \mathrm{cm}^{2}$ below $T_{\mathrm{C}} \sim 875 \mathrm{~K}$. The orderings of $\mathrm{Mn}$ and $\mathrm{Ho}$ ions occur at $75 \mathrm{~K}$ and $4.6 \mathrm{~K}$, respectively. Furthermore, the ferromagnetic ordering in $\mathrm{HoMnO}_{3}$ can be switched by an applied electric field via ME interactions. ${ }^{95}$ Recently, theoretical calculation predicts a huge electronic contribution to the ferroelectricity in $\mathrm{HoMnO}_{3}$ thin films with large strain, but so far it has not been confirmed experimentally. ${ }^{96}$ Actually, in this E-type AFM state, the magnetically driven spontaneous polarization was theoretically predicted to be as large as the values observed in the conventional perovskite ferroelectrics. ${ }^{97,98}$

\section{Orthorhombic $\mathrm{RMnO}_{3}$ with spiral spin order (SSO)}

The magnetic control of ferroelectricity in TMO was first reported in 2003 by Kimura et $\mathrm{al}^{7}{ }^{\mathrm{T}}$ TMO has a perovskite crystal structure with lattice parameters of $a=5.2931 \AA$, $b=5.8384 \AA$, and $c=7.4025 \AA{ }^{75}$ In this material, the breaking of spatial-inversion and time-reversal symmetries simultaneously occurs, and the ferroelectricity is induced by the inherent magnetic configuration, i.e., SSO. So far, dozens of magnetically driven multiferroic materials have been identified, and two types of SSO were revealed to be responsible for producing spontaneous polarization, as schematically illustrated in Figures 7(a) and 7(b). ${ }^{99,100}$ The corresponding mechanism of the multiferroicity can be generally understood using the inverse effect of the Dzyaloshinskii-Moriya (DM) interaction, $\boldsymbol{P} \propto-\boldsymbol{e}_{i, j} \times\left(\boldsymbol{S}_{i} \times \boldsymbol{S}_{j}\right)$, where $\boldsymbol{e}_{i, j}$ is the unit vector connecting the nearest-neighboring (NN) spins $\boldsymbol{S}_{i}$ and $\boldsymbol{S}_{j}{ }^{101}$

Typical materials with cycloidal spin ordering are TMO and $\mathrm{DyMnO}_{3}(\mathrm{DMO})$, in which a collinear sinusoidal antiferromagnetic spin ordering arises $\left(T_{\mathrm{N}}=41 \mathrm{~K}\right.$ for TMO and $39 \mathrm{~K}$ for DMO), and further cooling causes a $b c$-plane cycloidal (transverse-spiral) spin ordering with magnetically induced spontaneous polarization along the $c$ axis $\left(T_{\mathrm{C}}=28 \mathrm{~K}\right.$ for TMO and $19 \mathrm{~K}$ for DMO). ${ }^{102} \mathrm{~A}$ schematic drawing of the spin configuration and the temperature-dependent evolution of physical properties in TMO are presented in Figures $7(\mathrm{c})-7(\mathrm{e}){ }^{7}$ For the physics of bulk SSO multiferroic materials, three reviews are recommended. ${ }^{4,99,100}$

TMO thin films were synthesized and interesting ferromagnetic tendency was revealed. ${ }^{103-108}$ Detailed

(a) Cycloidal

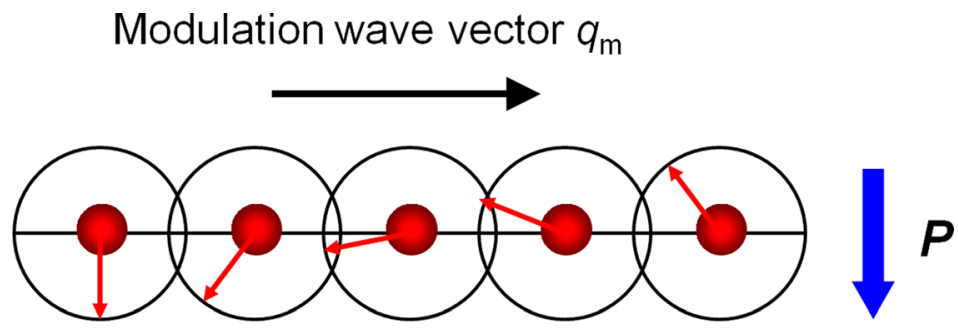

(b)
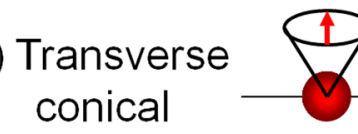

(c)

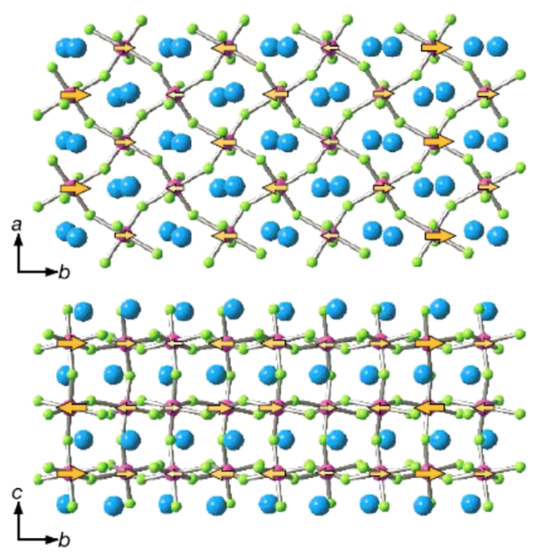

(d)

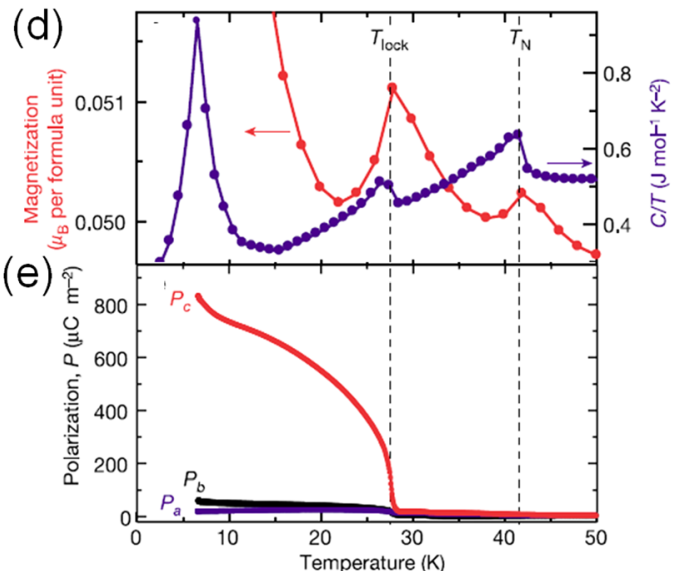

FIG. 7. Schematic drawings of (a) cycloidal and (b) transverse conical spin ordering. (c) Collinear sinusoidal spin ordering as $T_{\mathrm{C}}<T<T_{\mathrm{N}}$ (up), and spiral-spin-ordering as $T<T_{\mathrm{C}}$ in multiferroic $\mathrm{TbMnO}_{3}$. Temperature dependent magnetization and specific heat (d), and spontaneous polarization (e) along different axes in $\mathrm{TbMnO}_{3}$. Reprinted with permission from Kimura et al., Nature 426, 55 (2003). Copyright 2003 Nature Publishing Group. 


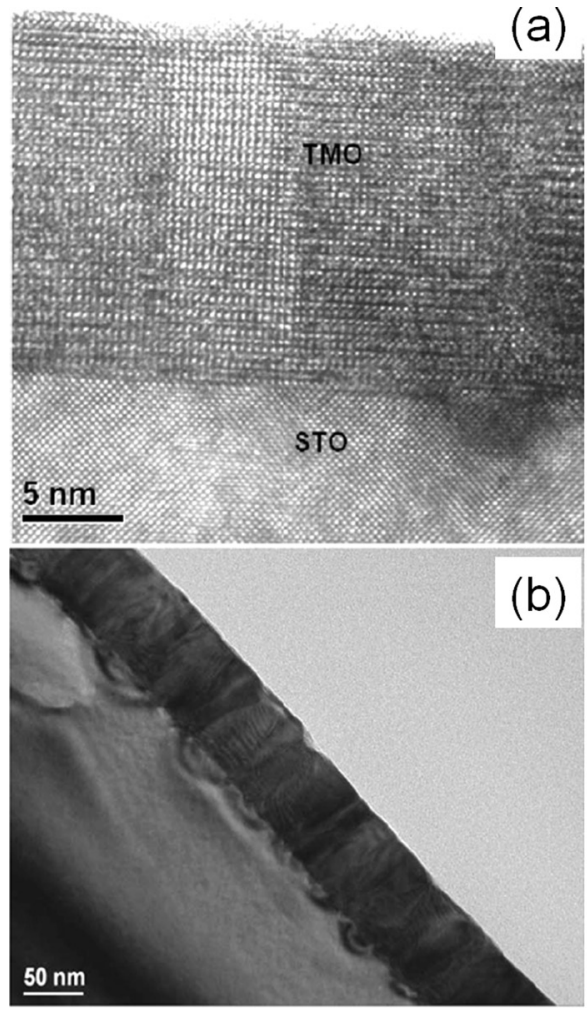

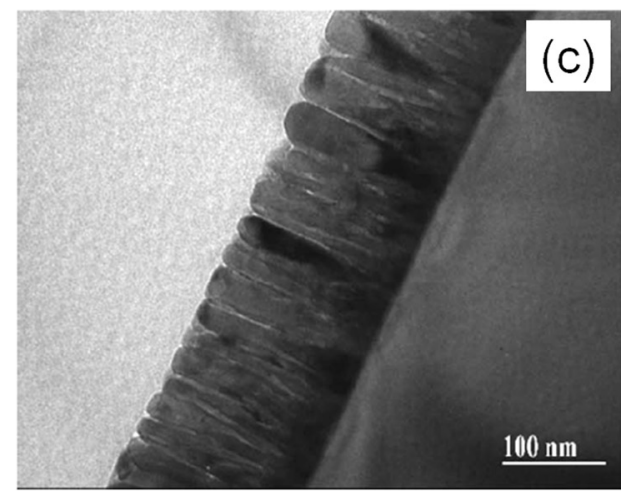

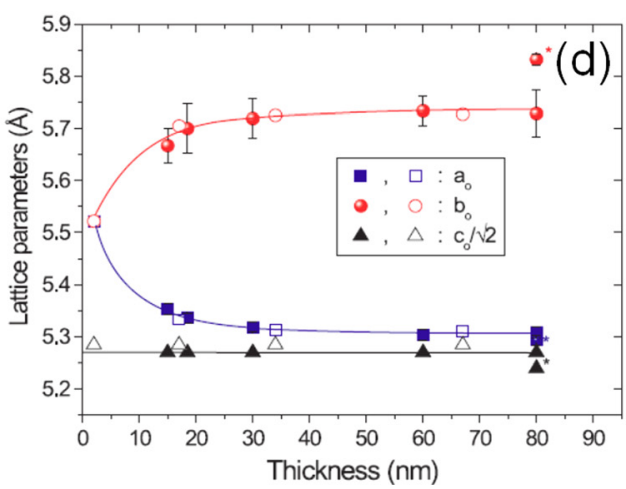

FIG. 8. Bright-field cross-section TEM images of $\mathrm{TMO} / \mathrm{SrTiO}_{3}$ thin films with different thicknesses (a) $17 \mathrm{~nm}$; (b) $67 \mathrm{~nm}$; and (c) $140 \mathrm{~nm}$. Reprinted with permission from Venkatesan et al., Phys. Rev. B 80, 214111 (2009). Copyright 2009 American Physical Society. (d) Evolution of lattice parameters with thickness. Adapted with permission from Daumont et al., J. Phys.: Condens. Matter 21, 182001 (2009). Copyright 2009 Institute of Physics. magnetometry, x-ray, and neutron scattering investigations confirmed that the emergent ferromagnetism in TMO thin films is an intrinsic property. ${ }^{106}$ Two possible mechanisms were proposed to be responsible for the emerged ferromagnetism: (i) strain-induced modulation of the competition between antiferromagnetic and ferromagnetic exchange interactions, ${ }^{108}$ and (ii) magnetic contribution from the domain walls. ${ }^{103}$ By combining $x$-ray photoemission measurements and $A b$ initio calculations, Rubi et al. revealed that the Mn $3 s$ splitting in TMO thin films is $0.3 \mathrm{eV}$ larger than that of the bulk counterpart. ${ }^{104}$ Detailed structural characterizations were also carried out on $\mathrm{TMO} / \mathrm{SrTiO}_{3}$ thin films. ${ }^{103,105,109}$ Very clear nanoscale domains were observed, and the crystalline structure transforms from the tetragonal phase to the orthorhombic one via a twinning process. The evolution of crystal structure with film thickness characterized using TEM and high-resolution x-ray diffraction is shown in Figure 8.

Although interesting ferromagnetism has been reported in TMO thin films, measuring the small spontaneous polarization in this type of spiral multiferroic materials remains challenging. Recently, the magnetically induced ferroelectric state was confirmed in TMO thin films grown on $\mathrm{YAlO}_{3}$ (001) substrates by using the second harmonic generate method, and the ferroelectricity can even exist in films as thin as $1.6 \mathrm{~nm} .{ }^{110}$ In DMO (Figure 9) and $\mathrm{GdMnO}_{3}$ thin films grown on $\mathrm{SrTiO}_{3}$ (001), the ferroelectric polarization was clearly demonstrated by both pyroelectric current method and hysteresis measurement, and large magnetic anisotropy and magnetic field induced polarization enhancement were also identified. ${ }^{111,112}$ In particular, large spontaneous polarization was reported in $\mathrm{GdMnO}_{3}$ thin films, which is probably due to a self-assembled nano-twinned domain structure. ${ }^{112}$ This result indicates that finely tuning the competing balance of multiple interactions is a promising route to explore novel spin-dictated multiferroicity in classic multiferroics. In addition, while the ground state of bulk orthorhombic YMO is E-type antiferromagnetic, a strain induced spiral spin ordering phase accompanied by spontaneous polarization was recently reported in thin films, and an intriguing memory effect was evidenced. ${ }^{113,114}$ The recent progresses of directly measuring the spontaneous polarization in these spiral multiferroic thin films open the possibility of integrating them into functional devices. Furthermore, a few recent works reported gigantic magnetically induced ferroelectric polarization and ME coupling in bulk crystals of

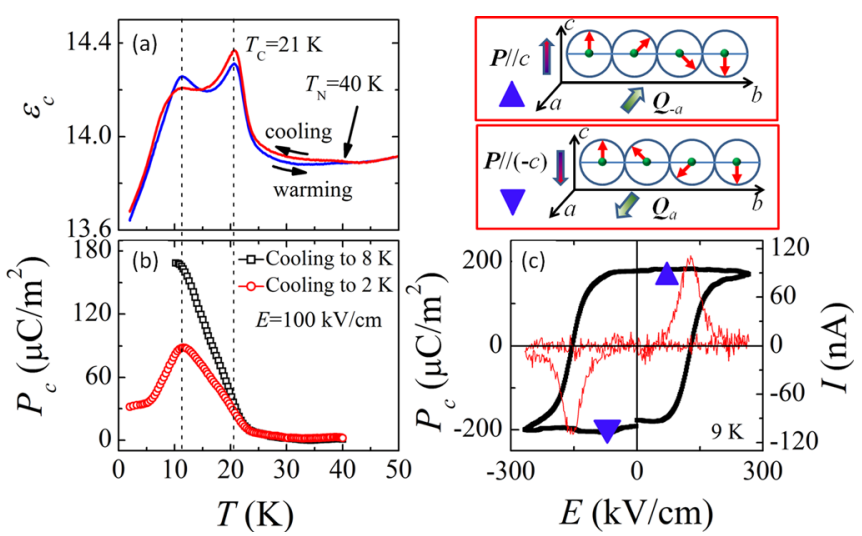

FIG. 9. Dielectric and ferroelectric properties of $\mathrm{DyMnO}_{3}$ thin films. Temperature dependence of $\varepsilon_{c}$ (a) and $P_{c}$ (b). (c) Ferroelectric hysteresis loop measured at $T=9 \mathrm{~K}$ without magnetic field during the cooling process. A sketch of the clockwise $\left(Q_{-a}\right)$ and counterclockwise $\left(Q_{a}\right)$ spiral spin orders is also presented. Adapted with permission from Lu et al., Sci. Rep. 3, 3374 (2013). Copyright 2013 Nature Publishing Group. 
$\mathrm{CaMn}_{7} \mathrm{O}_{12},{ }^{115} \mathrm{CaBaCo}_{4} \mathrm{O}_{7},{ }^{116}$ and $\mathrm{TbMnO}_{3}$ under pressure, ${ }^{117}$ which may motivate more thin film research.

\section{Other multiferroic thin films}

The search for more multiferroic materials with high performances has been fruitful, and along the process new physics was revealed. However, it is unrealistic to exhaust here all the related works in literature because of the space constraint. Instead, we focus here on one example: delafossite-type $\mathrm{Cu}_{M} \mathrm{O}_{2}(M=\mathrm{Cr}, \mathrm{Mn}, \mathrm{Fe}$, or Co). This class of materials has a two-dimensional (2D) triangular crystal structure, in which the $M$ oxide magnetic layers and the cuprous oxide layers are alternately packed along the $c$ axis. These 2D triangular antiferromagnets have received lots of interest due to the inherent multiferroicity caused by the frustrated spin configuration. ${ }^{118,119}$ It was demonstrated for $\mathrm{CuCrO}_{2}$ that ferroelectric polarization reversal could be finely tuned by both magnetic and electric fields. ${ }^{120}$ Recently, a few works on multiferroic $\mathrm{Cu} M \mathrm{O}_{2}$ thin films were reported, and measurements of optical absorption spectra were performed on these multiferroic thin films. ${ }^{121,122}$ Two optical transitions were identified and associated with $\mathrm{Cu} 3 d+\mathrm{O} 2 p \rightarrow \mathrm{Cu} 3 d_{\mathrm{z}}^{2}+4 s$ and $\mathrm{Cu} 3 d+\mathrm{O} 2 p \rightarrow M 3 d$, as shown in Figure 10. These results indicate that epitaxial thin films of 2D triangular ferromagnets involving magnetic and excitonic features are promising to realize some novel functionalities.

\section{Magnetism in multiferroic thin films}

In most well-studied multiferroic materials such as BFO and TMO, the antiferromagnetic spin order, which coexists

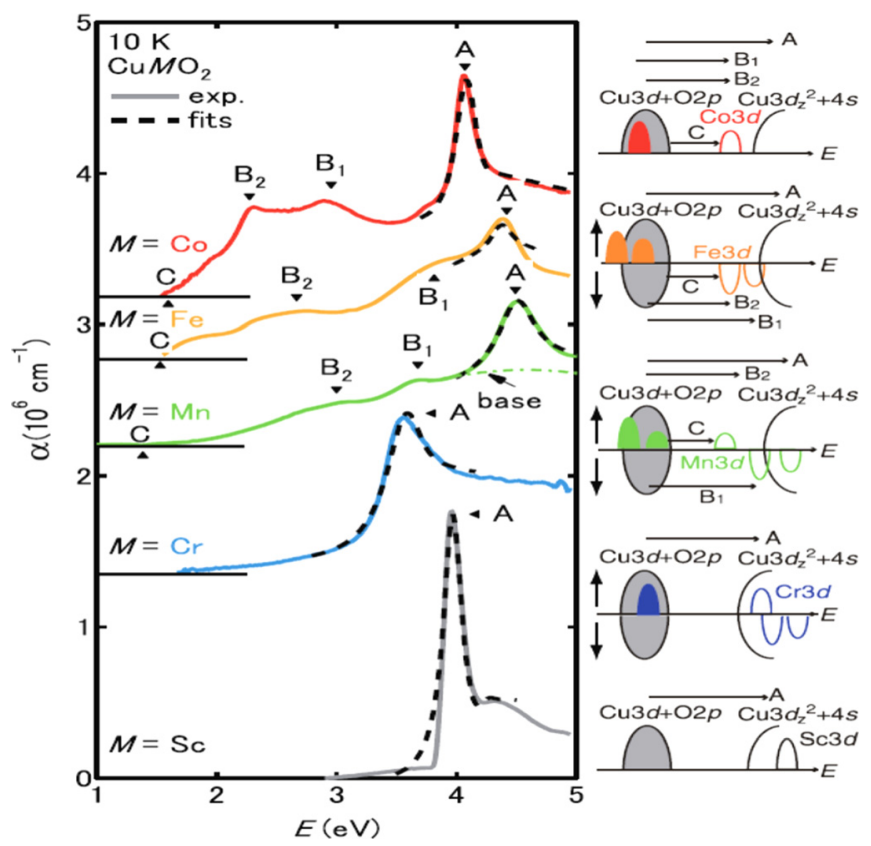

FIG. 10. Absorption spectra (solid lines) of $\mathrm{CuMO}_{2}(M=\mathrm{Sc}, \mathrm{Cr}, \mathrm{Mn}, \mathrm{Fe}$, and Co) thin films. The right panel represents a schematic drawing of the energy diagrams derived from first-principles calculations. Arrows indicate transitions corresponding to the observed absorption peaks. Reprinted with permission from Hiraga et al., Phys. Rev. B 84, 041411 (2011). Copyright 2011 American Physical Society. with ferroelectricity, does not give rise to any macroscopic magnetic moment. Canting of the AFM aligned spins by the DM interaction can give rise to weak FM. ${ }^{101}$ For multiferroic device applications, it is beneficial to enhance the magnetism in these materials and to achieve sizable spontaneous magnetism. In literature, there are several reported approaches of enhancing the magnetism in multiferroic oxide thin films. It is not a surprise that most of such works have focused on BFO.

Combining the angle and temperature dependent dichroic measurements and spectroscopy, Holcomb et al. observed that the antiferromagnetic phase in BFO epitaxial thin films is sensitive to the thickness and strain. ${ }^{123}$ Similar magnetic modulation was observed in $\mathrm{BFO} / \mathrm{LaAlO}_{3}$ thin films with large compressive strain, in which the saturated magnetism and coercivity of the highly strained phase are about six times larger than those of the relaxed one (Figure 11). ${ }^{124} \mathrm{~A}$ weak but definite spin-glass (SG) transition was evidenced in $\mathrm{BFO} /$ $\mathrm{LaAlO}_{3}$ thin films. ${ }^{124,125}$ Overall, strain can exert significant effects on the magnetic property of BFO thin films. But it should be noted that such glassy magnetism in thin BFO films would be too weak to be integrated into functional devices.

In another approach, magnetism in BFO was enhanced through structural and compositional modulations. ${ }^{126-130}$ When $\mathrm{Bi}^{3+}$ is substituted by a larger radius ion (such as $\mathrm{Ba}^{2+}$ ), the net magnetization can be notably enhanced due to the suppression of the long range spiral-spin-ordering in BFO. ${ }^{126}$ Furthermore, rare-earth doping is an effective way to enhance the magnetism in BFO, which can be associated
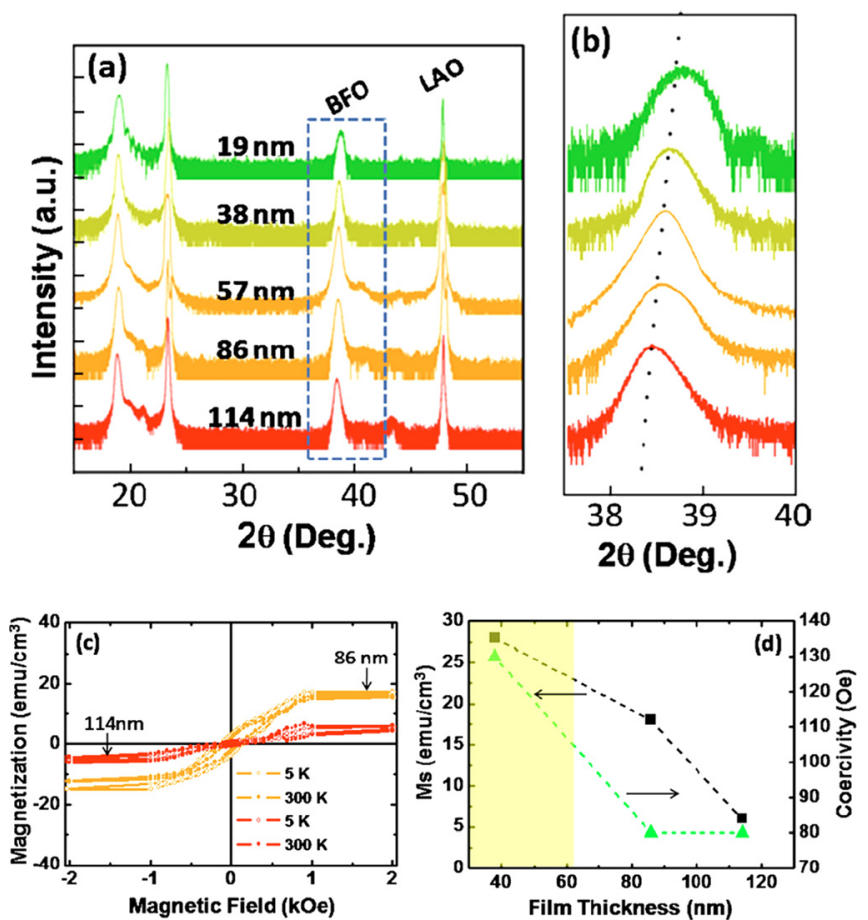

FIG. 11. (a) and (b) X-ray diffraction spectra of quasi-tetragonal phase $\mathrm{BFO} / \mathrm{LAO}$ films with various thicknesses. (c) Magnetic field dependence of magnetization for samples with different thicknesses measured under 300 and $5 \mathrm{~K}$. (d) Thickness dependence of room temperature saturated magnetization and coercivity. Reprinted with permission from Appl. Phys. Lett. 98, 242502 (2011). Copyright 2011 AIP Publishing LLC. 
with the crystal structure modulation as well as the magnetic coupling between rare-earth ions and $\mathrm{Fe}^{3+} .127,128$

Regarding the size effect, the period of spiral-spinordering in bulk BFO is about $62-64 \mathrm{~nm}$, so it would be of interest to see the magnetic variation as the size of BFO samples is scaled down into the nanometer regime. Indeed, a few groups reported ferromagnetism in nano-sized BFO, which was ascribed to the breakdown of the long-range spin order. ${ }^{129,130}$ These results also bear relevance to thin film and nanoscale device research, and new physics may emerge when the sample dimensions scale down to the characteristic length of spin order in BFO.

As aforementioned, interesting strain-engineered ferromagnetism has been observed in TMO thin films. ${ }^{104,106,107}$ In fact, similar modulation in magnetism was identified in YMO thin films as well. ${ }^{108,131}$ A large magnetic moment of $\sim 330 \mathrm{emu} / \mathrm{cm}^{3}=2.2 \mu_{\mathrm{B}} / \mathrm{Mn}$ at $15 \mathrm{~K}$ was observed in $\mathrm{BiMnO}_{3}$ films grown on $\mathrm{SrTiO}_{3}$ and the coercivity is $\sim 500 \mathrm{Oe}^{62} \mathrm{~A}$ later work found that the magnetic moment of $\mathrm{BiMnO}_{3}$ films can further increase to $\sim 3.2 \mu_{\mathrm{B}} / \mathrm{Mn}$ by growing on $\mathrm{SrTiO}_{3}{ }^{64}$ However, the magnetism usually appears quite weak in most multiferroic oxides. For example, a magnetic moment of only $\sim 0.075 \mu_{\mathrm{B}} / \mathrm{Mn}$ was found for YMO films grown on $\mathrm{YAlO}_{3}$ (001) substrates. ${ }^{76}$ It is worth of mentioning that a large ferromagnetic moment of $\sim 0.5 \mu_{\mathrm{B}} / \mathrm{Mn}$ with intrinsic exchange bias was evidenced in orthorhombic $\mathrm{LuMnO}_{3} / \mathrm{YAlO}_{3}$ (110) thin films. Combining the polarized neutron reflectometry technique, a possible ferromagnetic interlayer close to the substrate in the film was proposed to be the origin. ${ }^{132}$ These findings suggest that the multiferroic manganites can be promising candidates for new functional devices.

\section{DEVICES BASED ON MULTIFERROIC THIN FILMS}

\section{A. Resistive switching devices}

We start the discussion on multiferroic devices with resistive switching memories because of their simple twoterminal configuration and relatively straightforward operation mechanism. Since most multiferroic materials are excellent insulators, they can be directly applied in resistive switching devices for nonvolatile memory applications. Such devices usually show advantages of high density, high speed, and low power, and thus they are promising for next generation nonvolatile memory technology. Different from the switching operation of conventional dielectric materials where only the resistance can be switched between two states, namely, the high resistance state and the low resistance state, the resistive switching actions in multiferroic thin films could be accompanied by the concurrent modulation of the magnetic and ferroelectric properties, which, although has not been extensively explored so far, would drastically functionalize the devices.

Interesting switching effects were realized in BFO thin films: Wang et al. ${ }^{133}$ observed polarization-modulated conduction behaviors, including switchable diode effect and ferroelectric resistive switching, as shown in Figure 12. These interesting properties were attributed to the polarizationinduced variations of Schottky barrier. In addition, a novel ferroelectric-resistive non-volatile random access memory (RAM) consisting of BFO epitaxial thin films was reported. This device showed promising properties like excellent retention and high ON/OFF ratios. ${ }^{134}$

There are mainly two barrier-related mechanisms proposed to be responsible for the resistance switching observed in such multiferroic thin films. One is the polarization induced interface barrier, and the other is the charge injection induced interface barrier change, especially the electromigration of oxygen vacancies under the high electric field. However, which mechanism is dominant during a particular switching process is still under active debate. ${ }^{134-136}$ This is partially due to the diverse electric behaviours of BFO films which depend sensitively on the deposition conditions.

The well studied multiferroic materials YMO, TMO, and DMO were also used in memory devices, which show quite high ON/OFF ratios $\left(>10^{4}\right)$ and good retention $\left(>10^{5} \mathrm{~s}\right)$. The ferroelectricity in such films is much weaker than that of BFO. The mechanism of resistive switching in these thin films was ascribed to the rupture and formation of conductive filaments. ${ }^{137-139}$ For example, at the ON state, the carriers such as oxygen vacancies would be driven to form a certain pathway throughout the device, resulting a highly conductive path in the device and thereby a smaller resistance. On the other hand, for the OFF state, all those carriers are distributed randomly within the device, and there is no conductive channel, giving rise to a high resistance state.

\section{B. Ferroelectric/multiferroic tunnel junctions}

The above mentioned resistive-switching behaviour is often observed in oxide films with thickness of hundreds of
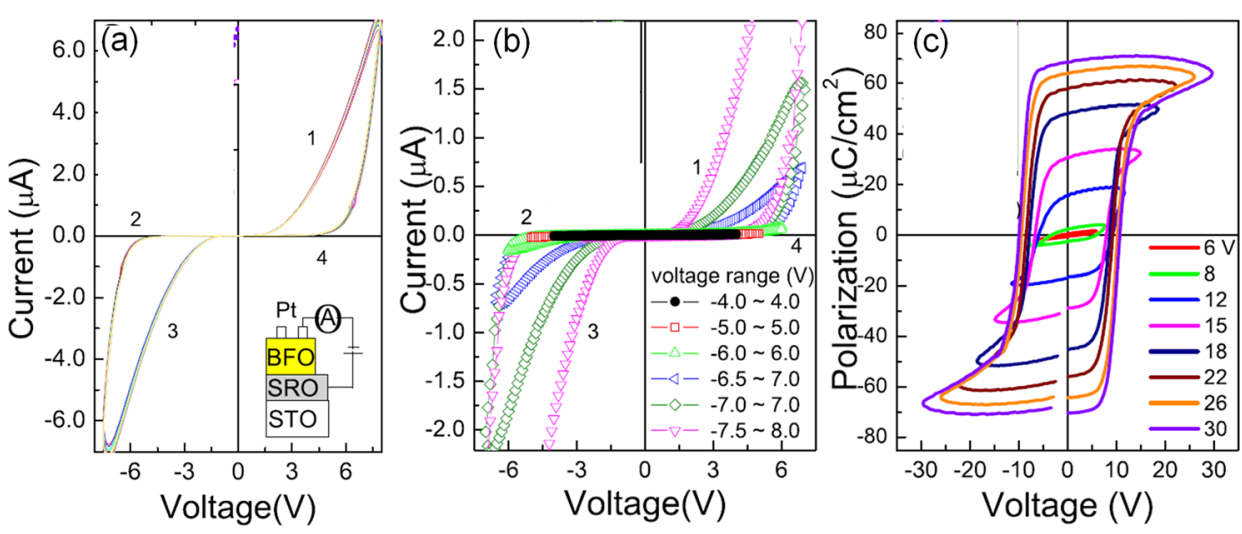

FIG. 12. (a) $I-V$ curves for BFO thin film. The schematic of the device structure is plotted in the inset. (b) $I-V$ curves measured on a virgin BFO thin film with different voltage sweeping ranges. The resistive switching behavior appears when the sweeping voltage is larger than $6 \mathrm{~V}$. (c) Ferroelectric hysteresis loops measured under different voltage ranges. Reprinted with permission from Appl. Phys. Lett. 98, 192901 (2011). Copyright 2011 AIP Publishing LLC. 
nanometers. In this thickness range, both the interface barrier and the ferroelectric itself contribute to the transport. However, when the ferroelectric layer thickness goes down to several nanometers, where electric tunnelling occurs, the polarization controlled interface barrier will become the dominant factor due to the large depolarization field. Based on this idea, the device concept of FTJ was proposed, ${ }^{140}$ which is composed of an ultra-thin FE layer sandwiched between two metal electrodes (M/FE/M).

Figure 13 shows the schematic band diagram of a FTJ device. ${ }^{140}$ Due to the charge screening effect, the reversal of polarization will change the charge distribution on the ferroelectric-metal interface and thus modify the tunnel barrier height the tunnelling electrons experience. Tunnelling electroresistance (TER) is thus defined as the ratio of resistance values corresponding to different barriers. Other effects such as the chemical bonding at the interface and the piezoinduced strain effect (Figure 13, right) could also contribute to TER.

The maintenance of the ferroelectricity in the ultrathin films is the main challenge of fabricating functional FTJs. Benefitting from the improvement of thin film deposition technology in recent years, ferroelectricity in ultrathin films ${ }^{141}$ and the related FTJs have been demonstrated in $\mathrm{BaTiO}_{3}, \mathrm{PbZr}_{0.2} \mathrm{Ti}_{0.8} \mathrm{O}_{3}$, and $\mathrm{BFO}$ ultrathin films. ${ }^{142-144}$ Different with the magnetic tunnel junctions, where the writing is accomplished through the current induced magnetic field, the states of a FTJ are set using an electric field. This all electronic operation means low energy consumption in FTJs, which is one of the main advantages of using FTJ as a non-volatile memory device. Other advantages include the fast writing speed as a result of the fast domain switching and the multi-level resistance states. ${ }^{142,145}$ However, the drawbacks are also obvious, including the poor retention and endurance performance of the device, which are a result of the unstable ferroelectric polarization in ultrathin films due to the size effect. These issues have been partially resolved by Wen et al. recently, who extended the common M/FE/M FTJs to $\mathrm{M} / \mathrm{FE} / \mathrm{S}$ (semiconductor) FTJs such as $\mathrm{Pt} / \mathrm{BaTiO}_{3} /$ $\mathrm{Nb}-\mathrm{SrTiO}_{3}$ as shown in Figure $14 .{ }^{146}$ It was found that by replacing the metal electrode with a semiconductor, TER could be greatly enhanced by two orders of magnitude, together with the improvement of the device retention and endurance. It was claimed that the simultaneous modulation of barrier height and barrier width by the polarization is responsible for this TER enhancement, and this breakthrough will surely inspire more future efforts in this research direction.

Recently, the rare-earth manganite TMO in hexagonal structure was also used as tunnel barrier, and giant ON/OFF ratio of resistance $(30000 \%)$ due to the switching of TMO polarization was observed. ${ }^{147}$ This value is comparable with those in classic ferroelectric tunnel junctions and, moreover, TMO is a magnetic material. This work might inspire more interests in exploring superior properties in multiferroic tunnel junctions with rare-earth manganite barriers.

\section{Multiferroic/ferromagnetic heterostructures}

Although multiple ferroic-orders with intimate cross coupling coexist in some single-phase multiferroic material, the net magnetization is small, in general. To overcome this disadvantage, constructing heterostructures of multiferroic/ ferromagnetic materials is promising to realize advanced spintronic devices. Recently, several groups successfully combined the multiferroic materials with ferromagnetic ones in thin film heterostructures, and very interesting phenomena were observed. ${ }^{148}$ For example, exchange bias and uniaxial magnetic anisotropy were studied in BFO, YMO, and TMO

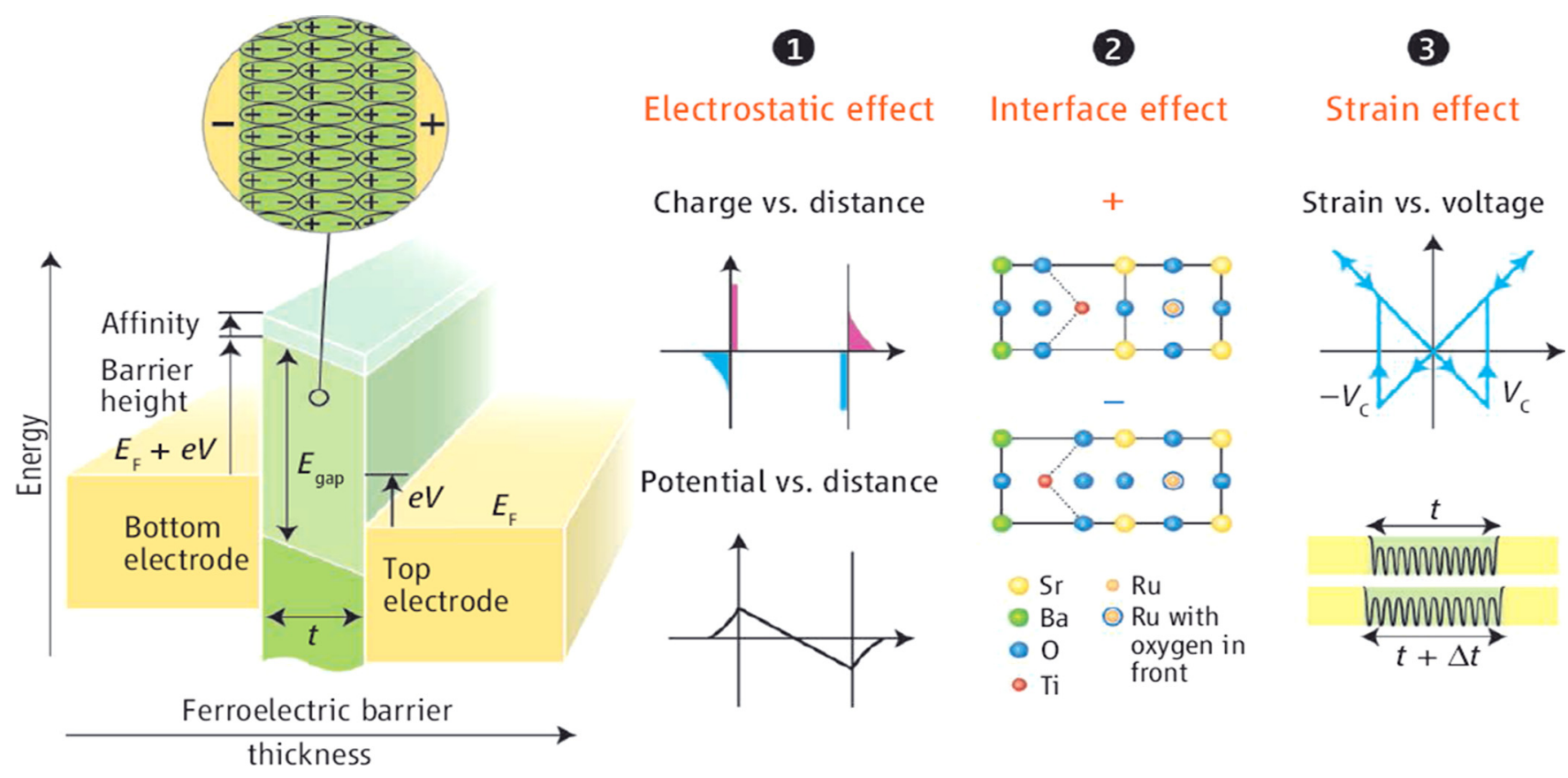

FIG. 13. Schematic diagram of a ferroelectric tunnel junction, which consists of two electrodes separated by a nanometer-thick ferroelectric barrier layer. $\left(E_{\text {gap }}\right.$ is the energy gap. $E_{\mathrm{F}}$ is the Fermi energy, $V$ is the applied voltage, $V_{\mathrm{c}}$ is the coercive voltage, $t$ is the barrier thickness, and $\delta t$ is the thickness variation under an applied field.) Reprinted with permission from E. Y. Tsymbal and H. Kohlstedt, Science 313, 181 (2006). Copyright 2006 AAAS. 
(a)

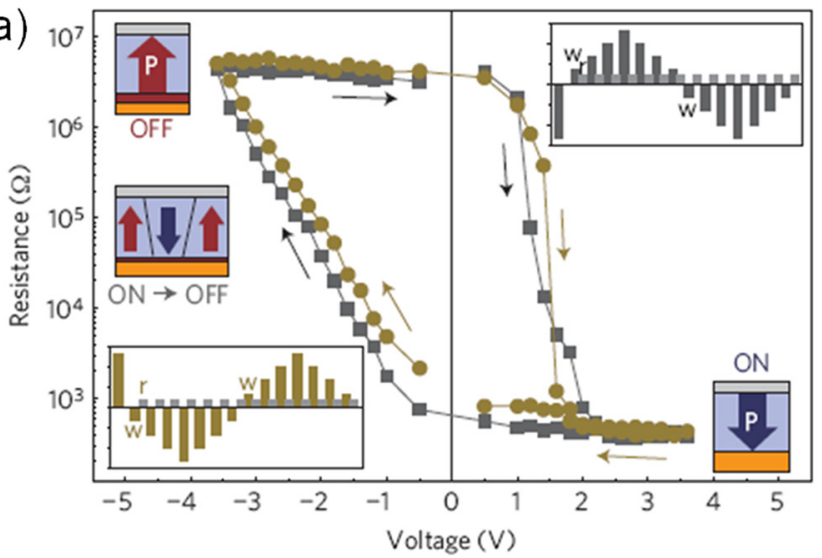

(b)

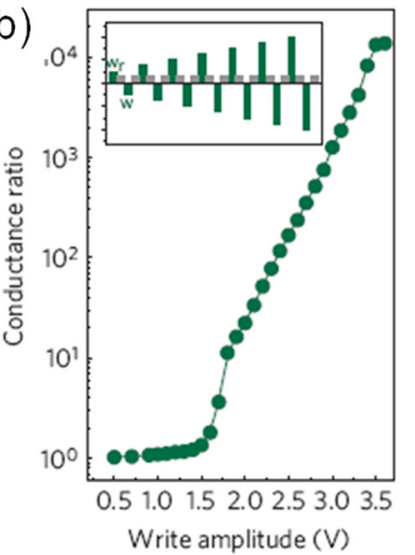

(c)

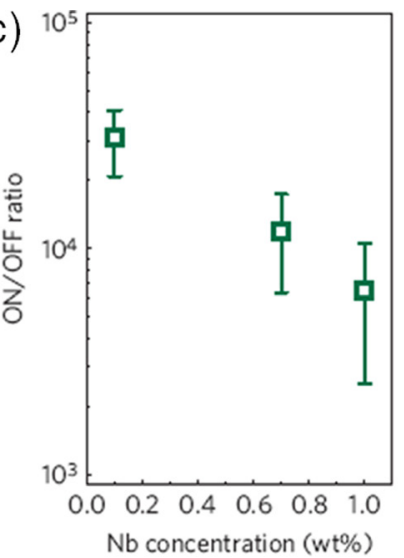

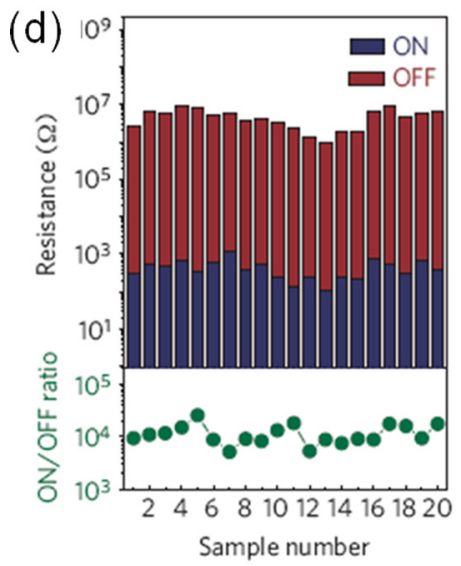

(e)

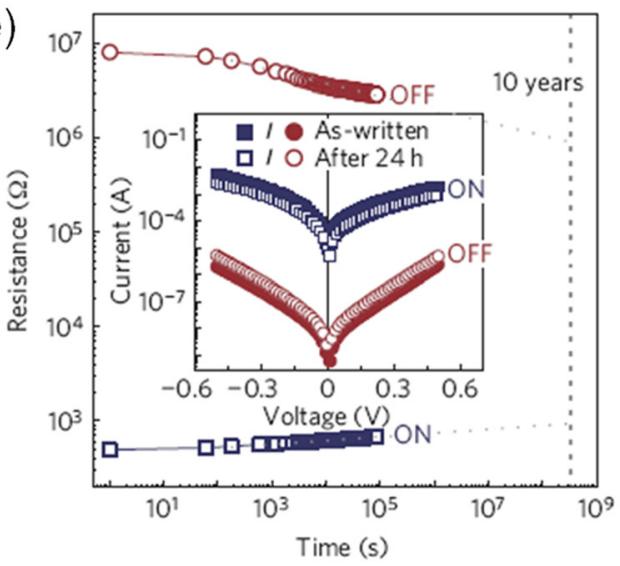

(f)

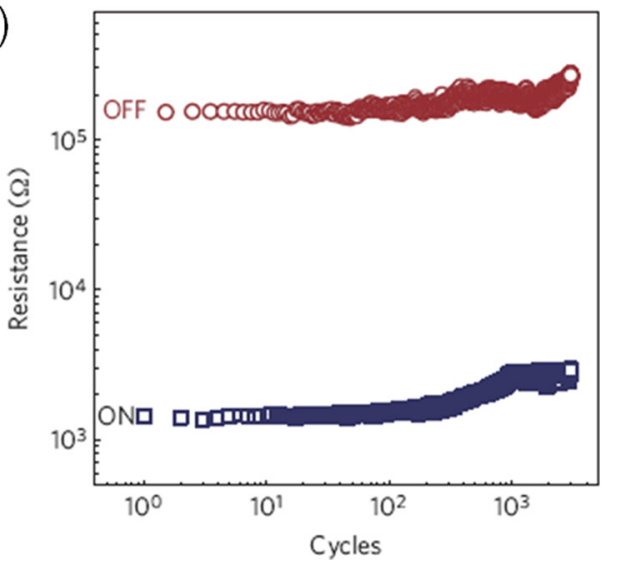

FIG. 14. (a) Resistance hysteresis loops measured on the $\mathrm{Pt} / \mathrm{BaTiO} / \mathrm{Nb}-\mathrm{SrTiO}_{3}$ heterostructure. The corresponding domain evolution is shown schematically in the bottom-right, top-left, and middle-left insets for the ON, OFF, and intermediate states, respectively. The testing pulse train is composed of write pulses (w) following a triangular profile between +3.6 and $-3.6 \mathrm{~V}$ and read pulses (r) of $+0.1 \mathrm{~V}$ following each write pulse. (b) Conductance ratio as a function of write amplitude. (c) ON/OFF ratio read at $+0.1 \mathrm{~V}$ as a function of $\mathrm{Nb}$ doping concentration in $\mathrm{Nb}^{\mathrm{SrTiO}} \mathrm{Sr}_{3}$ (d) ON and OFF state resistances (upper panel) and the corresponding ON/OFF ratios (bottom panel) of 20 different devices. (e) Retention properties. The inset shows current-voltage curves collected before and after the retention measurement. (f) Bipolar resistance switching properties. Reprinted with permission from Wen et al., Nat. Mater. 12, 617 (2013). Copyright 2013 Macmillan Publishers Limited.

based heterostructures. ${ }^{149-163}$ As a milestone in the research on electric field control of magnetism, Zhao and coworkers demonstrated that the antiferromagnetic domains of BFO can be switched simultaneously with the ferroelectric domains using an electric field. ${ }^{164}$ Ferromagnetic domains of the top $\mathrm{Co}_{0.9} \mathrm{Fe}_{0.1}$ layer can be electrically switched when it was deposited on the BFO thin film, which indicates a strong magnetic interaction at the interface between the AFM order in BFO and the FM order in CoFe (Figure 15). ${ }^{152,163}$ Recently, Allibe et al. reported interesting electrical control of giant magnetoresistance (GMR) in spin valves exchangebiased with BFO, which represents another important progress in the field. ${ }^{165}$

The first observation of the electric field control of exchange bias effect was reported in a Py/YMO heterostructure at a very low temperature of $2 \mathrm{~K}^{161}$ Exchange bias effects were later obtained in multiferroic $\mathrm{BiFeO}_{3} /$ $\mathrm{La}_{0.7} \mathrm{Sr}_{0.3} \mathrm{MnO}_{3}$ (LSMO) heterostructures without the need of heating to the Néel temperature of BFO, which can be further modulated by a gate electric field. ${ }^{156,157}$ A model was proposed to take into account the field-induced ionic displacements in BFO, and the modulation of the interatomic distance between the $\mathrm{Fe}$ and $\mathrm{Mn}$ ions presumably leads to the observed switching of exchange bias. Intriguingly, a recent structural investigation revealed that the field effect should be ascribed to the motions of oxygen ions at the interface. ${ }^{166}$

The magnetoelectric effect may enable the construction of novel device concepts like magnetoelectric RAMs (MeRAMs) which combines the magnetoelectric coupling with the interfacial exchange coupling between multiferroic and ferromagnetic layers. ${ }^{167}$ In MeRAM, a voltage is used to switch the exchange coupling across the interface and in turn the magnetization of the ferromagnetic layer. This voltage mode of operation supposedly consumes much less energy than the current mode used in devices like MRAMs (magnetic RAMs). The mechanism of the exchange bias effects in such multiferroic/ferromagnetic heterostructures is still controversial. ${ }^{168-170}$ We would refer the readers to a few recent reviews dedicated to this topic of magnetoelectric effects. ${ }^{4,41,45}$ Nevertheless, these phenomena pave the way for integrating multiferroics into modern memory devices with potential high performances.

\section{Multiferroic/superconducting heterostructures}

Multiferroic/superconducting heterostructures, such as BFO- $\mathrm{YBa}_{2} \mathrm{Cu}_{3} \mathrm{O}_{7}$ (YBCO) heterostructures, are interesting 
(a)

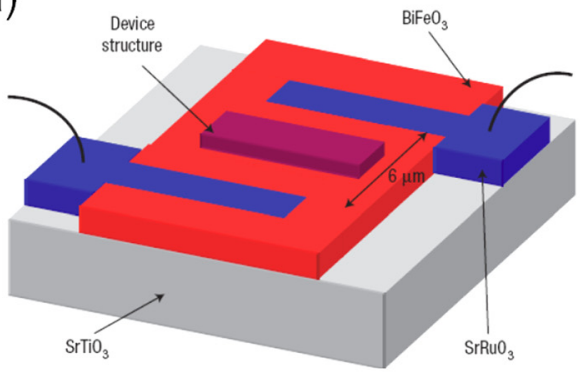

(b)

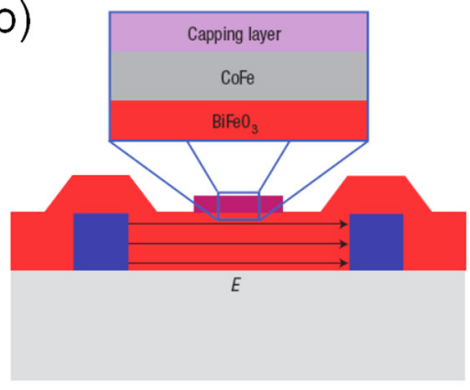

(c)

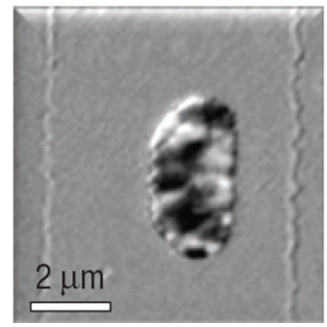

(d)

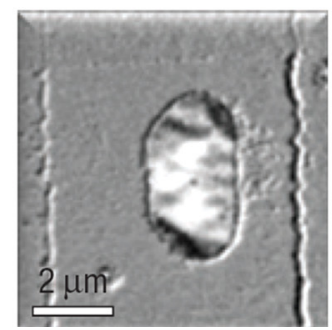

(e)

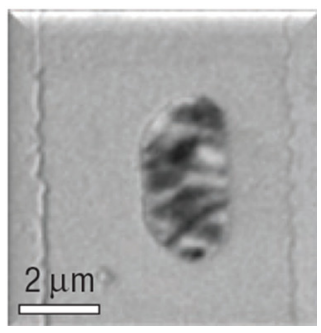

FIG. 15. (a) and (b) Schematics of the multiferroic-ferromagnetic device structure. Magnetic state of the $\mathrm{CoFe}$ layer was obtained using the $\mathrm{x}$-ray magnetic circular dichroism (XMCD)photoemission electron microscopy (PEEM) (white and black correspond to different magnetic polarities). (c) Original state; (d) domain state after the bottom layer of BFO was electrically switched; and (e) the BFO layer was switched back to the original state. Reprinted with permission from Chu et al., Nat. Mater. 7, 478 (2008). Copyright 2008 Nature Publishing Group. in considering the possible interactions between the superconducting order with the multiple ferroic orders at the interface. Indeed, a variety of fascinating properties have been evidenced in YBCO-based junctions. ${ }^{171-175}$ Epitaxial growth of $\mathrm{BFO} / \mathrm{YBCO}$ heterostructure can be realized due to the compatible lattice structure in the two materials with a lattice mismatch of $\sim 3 \%$. Interfacial couplings in such heterostructures may drastically upset the delicate balance of the competing interactions among charges, spins, orbitals, and lattice, which can potentially present a wide range of emergent phenomena.

Recently, high-quality BFO/YBCO heterostructure and its transport properties were reported. Interestingly, an enhancement of the interface barrier induced by the superconducting gap of YBCO was observed (Figure 16). ${ }^{176}$ Moreover, the multiferroic field effect was reported in similar $\mathrm{BFO} / \mathrm{YBCO}$ heterostructures, in which the superconducting condensate was modulated at nanoscale via the field effect. ${ }^{177}$ We should note here that there has been no report on any theoretical framework which can guide the experiments on such multiferroic/superconducting heterostructures,
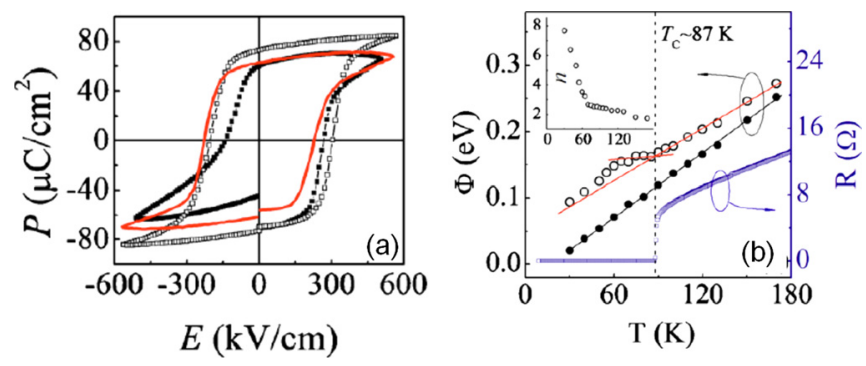

FIG. 16. (a) Ferroelectric hysteresis loops for the BFO/YBCO heterostructure measured at $77 \mathrm{~K}$ (open squares) and $300 \mathrm{~K}$ (solid squares). (b) Temperature dependence of the Schottky barrier height and resistance for the $\mathrm{BFO} / \mathrm{YBCO}$ heterostructure. The inset shows the $T$-dependent refractive index $n$. For a comparison, the $P-E$ loop (red line in (a)) and $\Phi(T)$ (solid dots in (b)) of a $\mathrm{BiFeO}_{3} / \mathrm{SrRuO}_{3}$ heterostructure were also presented. Reprinted with permission from Appl. Phys. Lett. 97, 252905 (2010). Copyright 2010 AIP Publishing LLC. and data interpretation remains challenging. More future efforts are needed in order to elucidate the interface physics in such multiferroic/superconducting heterostructures.

\section{E. Photovoltaic effect in multiferroic thin films}

Recently, interesting PV effects were observed in BFO thin films with striped domains, and the produced voltage was significantly higher than the bandgap (Figure 17). ${ }^{53}$ To understand this novel PV effect in BFO thin films, a ferroelectric-domain-wall-based model was proposed. ${ }^{52,53}$ In the ferroelectric domain walls, the built-in potential steps caused by the component of polarization can efficiently separate the photo-generated electrons and holes. The accumulation of the electrons/holes in the domain walls would build up a net electric voltage across the sample, leading to the observed open circuit photovoltage. This model can well explain the $\mathrm{PV}$ effect in BFO thin films with periodic domain structures.

On the other hand, a few groups reported PV effects in BFO thin films without striped domain and even in bulk monodomain BFO single crystals. ${ }^{63,135,178-180}$ Furthermore, PV effects were also observed in traditional ferroelectrics without a regular domain configuration. ${ }^{181-183}$ Concerning the role of domain walls on the abnormal PV effect in BFO thin films, Bhatnagar et al. performed temperature dependent $\mathrm{PV}$ effect measurements, proved that the bulk PV effect is the origin, ${ }^{184}$ and revealed an interesting persistent PV effect in strained BFO. ${ }^{185}$ After comparing with the traditional PV effects in $p-n /$ Schottky junctions, Yuan and Wang proposed a model based on the ferroelectric polarization modified depletion region to explain the PV effects evidenced in ferroelectric materials. ${ }^{186}$ In this model, the depletion region is modified when the polarization is switched, and thus the directional dependence of PV voltage and current on the polarization can be understood.

It is generally observed that the bulk photovoltaic effect is switchable with the polarization reversal, ${ }^{135}$ which is proposed to be due to the polarization induced transition between Schottky and Ohmic contacts and the electromigration of 
(a)

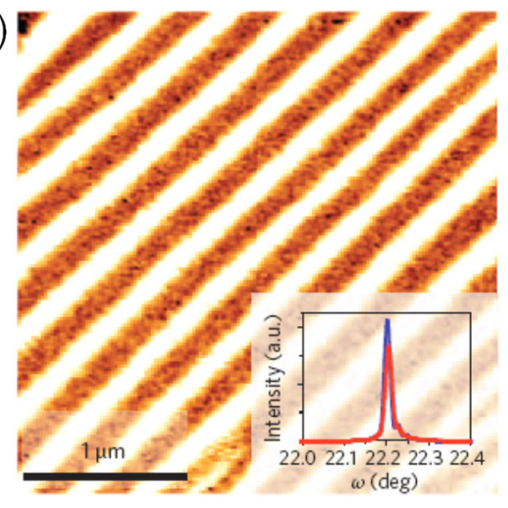

(c)
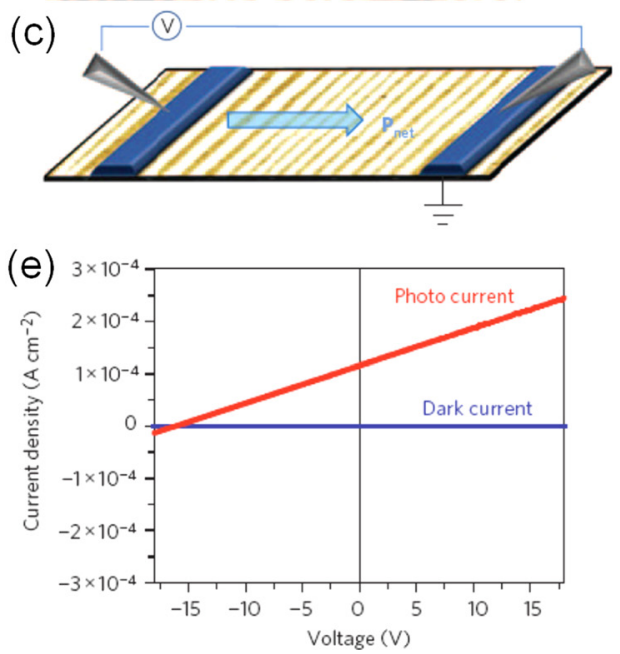

(b)

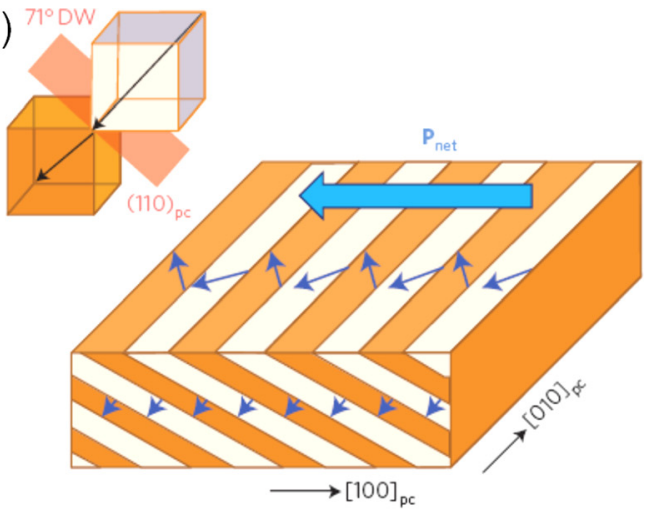

(d)

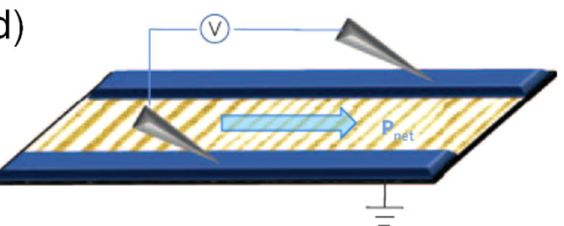

(f)

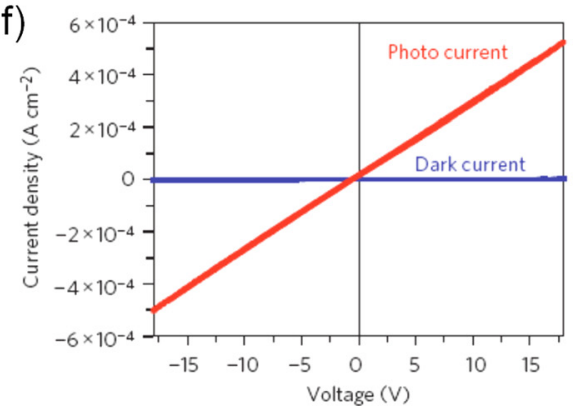

FIG. 17. (a) PFM image for periodic arrays of $71^{\circ}$ domain walls. The inset shows the X-ray rocking curves along two orthogonal crystal axes. (b) Schematic drawing of the $71^{\circ}$ domain arrays. The arrows indicate different components of ferroelectric polarization. The large arrow indicates the net polarization. Also shown are the schematic drawings of two different measurement configurations: parallel to the domain walls (c) and perpendicular to the domain walls (d), as well as the corresponding $I-V$ curves measured perpendicular (e) or parallel (f) to the domain walls. Reprinted with permission from Yang et al., Nat. Nanotechnol. 5, 143 (2010). Copyright 2010 Macmillan Publishers Limited. defects such as oxygen vacancies. ${ }^{187}$ Based on this phenomenon, a new type of non-destructive FeRAM device has been proposed recently by Guo et al. ${ }^{188}$ The basic idea is to use the short circuit current $\left(I_{\mathrm{sc}}\right)$ or open circuit voltage $\left(V_{\mathrm{oc}}\right)$ to sense the direction of the polarization. Figure 18 shows the prototype device structure, where a positive (negative) $V_{\text {oc }}$ corresponds to the up (down) polarization. $V_{\mathrm{oc}}$ as well as the polarization state could be read once there is light illumination on the device. This is a non-destructive operation process without any electric energy consumption, which represents an advantage compared with the traditional FeRAM devices.

Regarding to the light-induced effect, Kundys et al. recently reported an interesting phenomenon that the size of BFO crystal can be modulated by light illumination. ${ }^{189}$ In this experiment, a bulk mono-domain BFO crystal was used, and a positive size change was observed when the sample was illuminated by light. The observation of photostriction indicates the possibility of realizing mechanical, magnetic, electric, and optical functionalities in monolithic multifunctional devices.

\section{FINAL REMARKS AND OUTLOOKS}

In Secs. I-III, we used several material systems to highlight not only the groundbreaking achievements but also some grand challenges in applying multiferroic materials in thin-film-based technologies. These challenges are related to issues such as excessive leakage current, weak magnetism, and elusive coupling mechanism between various ferroic orders. Complementary experimental and theoretical efforts are needed to address the materials challenges on composition and defect controls, substrate and strain engineering, as well as post-growth processing and patterning. In order to guide the thin film growth, advanced structural characterization and spectroscopic tools should be developed to enable nanoscale imaging and measurements. (a)

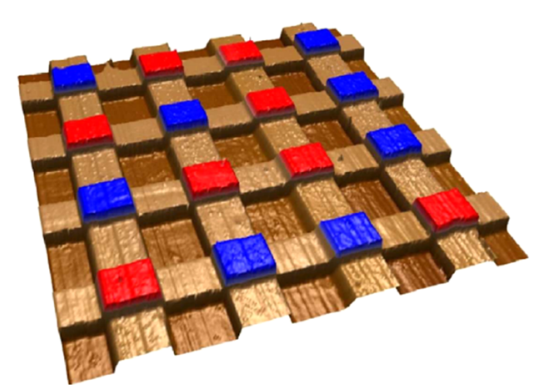

(b)

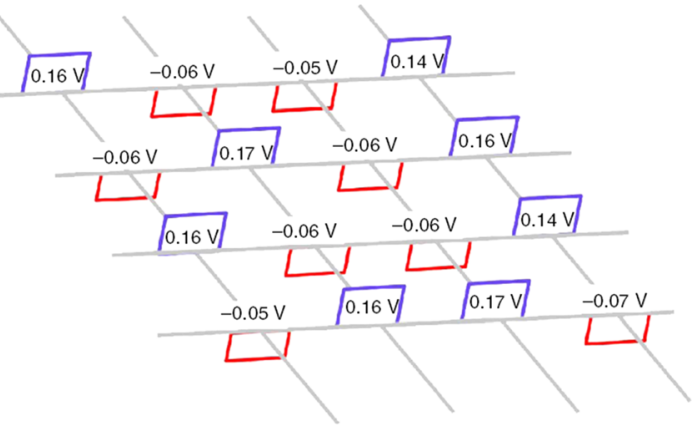

FIG. 18. Performance of a prototype 16-cell ferroelectric memory with the cross-bar architecture. (a) Topography of the device with preset polarization direction. Blue: polarization up, red: polarization down. (b) $V_{\mathrm{oc}}$ of all 16 cells are indicated. These were measured under $20 \mathrm{~mW} / \mathrm{cm}^{2}$ light illumination. Adapted with permission from Guo et al., Nat. Commun. 4, 1990 (2013). Copyright 2013 Nature Publishing Group. 
Discovering new multiferroic materials remains as the top priority in defining the future research directions. On the one hand, in Bi-based multiferroic materials, both ferroelectric and magnetic phase transitions can be higher than room temperature, and the polarization can reach the values found in classic ferroelectric materials. However, the magnetoelectric coupling is often very weak in these materials. On the other hand, in the multiferroics with spin origin, strong magnetoelectric effects have been observed due to the strong coupling between the ferroelectric and magnetic orders. But these materials generally show very low $\mathrm{T}_{\mathrm{C}}$, and the spontaneous polarization is about four orders of magnitude smaller than those of classic ferroelectrics. These drawbacks observed in the existing multiferroic materials plague the potential applications, which in turn motivate researchers to look for new high-performance materials.

In recent years, much research attention has been focused on multiferroic thin films and heterostructures, and indeed significant progresses have been made, and new device concepts were proposed and realized. Nevertheless, more efforts are desired to effectively bridge the fundamental research and technical applications. For instance, although combining ferroelectricity and spintronics in ME devices provides the possibility of nonvolatile memories with ultralow energy consumption, there has been no demonstration of reliable exchange-coupled multiferroic heterostructure operating at room temperature with high endurance and retention. Furthermore, more attempts are needed to advance domain engineering to either make mono-domain films to reproduce/improve the bulk-like physical properties or control domain structures to render novel functionalities in sensing and photovoltaic devices. Continuously improving the switching capability of ultrathin multiferroic films and elucidate the mechanism on the nanoscale are critical for device applications like ferroelectric/multiferroic tunneling junctions and nonvolatile memories. The dimensions of modern memory devices are often within the regime of tens of nm, but little is know how the multiferroic materials will behave at such small scales. Another important research front is compositional and structural interface/surface engineering on the atomic scale, which again is very challenging and remains largely unexplored for many multiferroics-based heterostructures. We believe that more significant breakthroughs in the field of multiferroic thin films and heterostructure devices will be witnessed in the near future.

\section{ACKNOWLEDGMENTS}

This work was supported by the Natural Science Foundation of China (Grant Nos. 11104090 and 11374112), and the King Abdullah University of Science and Technology (KAUST).

\footnotetext{
${ }^{1}$ R. Ramesh and N. A. Spaldin, Nat. Mater. 6, 21 (2007).

${ }^{2}$ N. A. Spaldin and M. Fiebig, Science 309, 391 (2005).

${ }^{3}$ S. W. Cheong and M. Mostovoy, Nat. Mater. 6, 13 (2007).

${ }^{4}$ K. F. Wang, J. M. Liu, and Z. F. Ren, Adv. Phys. 58, 321 (2009).

${ }^{5}$ I. E. Dzyaloshinskii, Sov. Phys. - JETP 10, 628 (1960).
}

${ }^{6}$ J. van den Boomgaard, D. R. Terrell, R. A. J. Born, and H. F. J. I. Giller, J. Mater. Sci. 9, 1705 (1974).

${ }^{7}$ T. Kimura, T. Goto, H. Shintani, K. Ishizaka, T. Arima, and Y. Tokura, Nature 426, 55 (2003).

${ }^{8}$ J. Wang, J. B. Neaton, H. Zheng, V. Vagarajan, S. B. Ogale, B. Liu, D. Viehland, V. Vaithyanathan, D. G. Schlom, U. V. Waghmare, N. A. Spaldin, K. M. Rabe, M. Wuttig, and R. Ramesh, Science 299, 1719 (2003).

${ }^{9}$ D. Khomskii, Physics 2, 20 (2009).

${ }^{10}$ N. A. Hill, J. Phys. Chem. B 104, 6694 (2000).

${ }^{11}$ J. M. Rondinelli and N. A. Spaldin, Adv. Mater. 23, 3363 (2011).

${ }^{12}$ C. Michel, J. M. Moreau, G. D. Achenbac, R. Gerson, and W. J. James, Solid State Commun. 7, 701 (1969).

${ }^{13}$ J. D. Bucci, B. K. Robertson, and W. J. James, J. Appl. Crystallogr. 5, 187 (1972)

${ }^{14}$ J. R. Teague, R. Gerson, and W. J. James, Solid State Commun. 8, 1073 (1970).

${ }^{15}$ Y. F. Tian, O. I. Lebedev, V. V. Roddatis, W. N. Lin, J. F. Ding, S. J. Hu, S. S. Yan, and T. Wu, Appl. Phys. Lett. 104, 152404 (2014).

${ }^{16}$ C. A. F. Vaz, J. Hoffman, C. H. Anh, and R. Ramesh, Adv. Mater. 22, 2900 (2010).

${ }^{17}$ Y. H. Lee, J. M. Wu, and C. H. Lai, Appl. Phys. Lett. 88, 042903 (2006).

${ }^{18}$ J. Schwarzkopf and R. Fornari, Prog. Cryst. Growth Charact. Mater. 52, 159 (2006).

${ }^{19}$ L. W. Martin, Y. H. Chu, and R. Ramesh, Mater. Sci. Eng., R 68, 89 (2010).

${ }^{20}$ D. G. Schlom, J. H. Haeni, J. Lettieri, C. D. Theis, W. Tian, J. C. Jiang, and X. Q. Pan, Mater. Sci. Eng., B 87, 282 (2001).

${ }^{21}$ K. J. Choi, M. Biegalski, Y. L. Li, A. Sharan, J. Schubert, R. Uecker, P. Reiche, Y. B. Chen, X. Q. Pan, V. Gopalan, L. Q. Chen, D. G. Schlom, and C. B. Eom, Science 306, 1005 (2004).

${ }^{22}$ R. J. Zeches, M. D. Ressell, J. X. Zhang, A. J. Hatt, Q. He, C.-H. Yang, A. Kumar, C. H. Wang, A. Melville, C. Adamo, G. Sheng, Y.-H. Chu, J. F. Ihlefeld, R. Erni, C. Ederer, V. Gopalan, L. Q. Chen, D. G. Schlom, N. A. Spaldin, L. W. Martin, and R. Ramesh, Science 326, 977 (2009).

${ }^{23}$ J. X. Zhang, Q. He, M. Trassin, W. Luo, D. Yi, M. D. Rossell, P. Yu, L. You, C. H. Wang, C. Y. Kuo, J. T. Heron, Z. Hu, R. J. Zeches, H. J. Lin, A. Tanaka, C. T. Chen, L. H. Tjeng, Y.-H. Chu, and R. Ramesh, Phys. Rev. Lett. 107, 147602 (2011).

${ }^{24}$ Q. He, Y.-H. Chu, J. T. Heron, S. Y. Yang, W. I. Liang, C. Y. Kuo, H. J. Lin, P. Yu, C. W. Liang, R. J. Zeches, W. C. Kuo, J. Y. Juang, C. T. Chen, E. Arenholz, A. Scholl, and R. Ramesh, Nat. Commun. 2, 225 (2011).

${ }^{25}$ Z. Chen, Z. Luo, C. Huang, Y. J. Qi, P. Yang, L. You, C. Hu, T. Wu, J. Wang, C. Gao, T. Sritharan, and L. Chen, Adv. Funct. Mater. 21, 133 (2011).

${ }^{26}$ J. X. Zhang, R. J. Zeches, Q. He, Y. H. Chu, and R. Ramesh, Nanoscale 4, 6196 (2012).

${ }^{27}$ J. Zhang, X. Ke, G. Guo, J. Seidel, B. Xiang, P. Yu, W. I. Liang, A. M. Minor, Y.-H. Chu, G. V. Tendeloo, X. B. Ren, and R. Ramesh, Nat. Commun. 4, 2768 (2013).

${ }^{28}$ J. C. Yang, Q. He, S. J. Suresha, C. Y. Kuo, C. Y. Peng, R. C. Haislmaier, M. A. Motyka, G. Sheng, C. Adamo, H. J. Lin, Z. Hu, L. Chang, L. H. Tjeng, E. Arenholz, N. J. Podraza, M. Bernhagen, R. Uecker, D. G. Schlom, V. Gopalan, L. Q. Chen, C. T. Chen, R. Ramesh, and Y.-H. Chu, Phys. Rev. Lett. 109, 247606 (2012).

${ }^{29}$ G. Catalan and J. F. Scott, Adv. Mater. 21, 2463 (2009).

${ }^{30} \mathrm{~T}$. Atou, H. Chiba, K. Ohoyama, Y. Yamaguchi, and Y. Syono, J. Solid State Chem. 145, 639 (1999).

${ }^{31}$ M. Gajek, M. Bibes, A. Barthélémy, K. Bouzehouane, S. Fusil, M. Varela, J. Fontcuberta, and A. Fert, Phys. Rev. B 72, 020406 (2005).

${ }^{32}$ M. Murakami, S. Fujino, S. H. Lim, C. J. Long, L. G. Salamanca-Riba, M. Wuttig, I. Takeuchi, V. Nagarajan, and A. Varatharajan, Appl. Phys. Lett. 88, 152902 (2006).

${ }^{33}$ B. Lorenz, Y. Wang, Y. Sun, and C. Chu, Phys. Rev. B 70, 212412 (2004).

${ }^{34}$ J. H. Lee, P. Murugavel, D. Lee, T. W. Noh, Y. Jo, M. H. Jung, K. H. Jang, and J. G. Park, Appl. Phys. Lett. 90, 012903 (2007).

${ }^{35}$ B. Veličkov, V. Kahlenberg, R. Bertram, and M. Bernhagen, Z. Kristallogr. 222, 466 (2007).

${ }^{36}$ J. M. Phillips, J. Appl. Phys. 79, 1829 (1996).

${ }^{37}$ J. H. Lee and K. M. Rabe, Phys. Rev. Lett. 104, 207204 (2010).

${ }^{38}$ W. Prellier, M. P. Singh, and P. Murugavel, J. Phys.: Condens. Matter 17, R803 (2005). 
${ }^{39}$ L. W. Martin, S. P. Crane, Y. H. Chu, M. B. Holcomb, M. Gajek, M. Huijben, C. H. Yang, N. Balke, and R. Ramesh, J. Phys.: Condens. Matter 20, 434220 (2008).

${ }^{40}$ R. Thomas, J. F. Scott, D. N. Bose, and R. S. Katiyar, J. Phys.: Condens. Matter 22, 423201 (2010).

${ }^{41}$ J. Ma, J. M. Hu, Z. Li, and C. W. Nan, Adv. Mater. 23, 1062 (2011).

${ }^{42}$ S. Seki, T. Kurumaji, S. Ishiwata, H. Matsui, H. Murakawa, Y. Tokunaga, Y. Kaneko, T. Hasegawa, and Y. Tokura, Phys. Rev. B 82, 064424 (2010).

${ }^{43}$ Y.-H. Chu, L. W. Martin, M. B. Holcomb, and R. Ramesh, Mater. Today 10, 16 (2007).

${ }^{44}$ F. Zavaliche, S. Y. Yang, T. Zhao, Y. H. Chu, M. P. Cruz, C. B. Eom, and R. Ramesh, Phase Transitions 79, 991 (2006).

${ }^{45}$ J. T. Heron, D. G. Schlom, and R. Ramesh, Appl. Phys. Rev. 1, 021303 (2014)

${ }^{46}$ Y. H. Chu, Q. He, C. H. Yang, P. Yu, L. W. Martin, P. Shafer, and R. Ramesh, Adv. Mater. 19, 2662 (2007).

${ }^{47}$ Y. H. Chu, Q. He, C. H. Yang, P. Yu, L. W. Martin, P. Shafer, and R. Ramesh, Nano Lett. 9, 1726 (2009).

${ }^{48}$ Y. H. Chu, Q. Zhang, L. W. Martin, M. P. Cruz, P. L. Yang, G. W. Pabust, F. Zavaliche, S. Y. Yang, J. X. Zhang, L. Q. Chen, D. G. Schlom, I. N. Lin, T. B. Wu, and R. Ramesh, Adv. Mater. 18, 2307 (2006).

${ }^{49}$ H. W. Jang, D. Ortiz, S. H. Beak, C. M. Folkman, R. R. Das, P. Shafer, Y. Chen, C. T. Nelson, X. Q. Pan, R. Ramesh, and C. B. Eom, Adv Mater. 21, 817 (2009).

${ }^{50}$ N. Balke, S. Choudhury, S. Jesse, M. Huijben, Y. H. Chu, A. P. Baddorf, L. Q. Chen, R. Ramesh, and S. V. Kalinin, Nat. Nanotechnol. 4, 868 (2009).

${ }^{51}$ J. Seidel, L. W. Martin, Q. He, Q. Zhang, Y. H. Chu, A. Rother, M. E. Hawkridge, P. Maksymovych, P. Yu, M. Gajek, N. Balke, S. V. Kalinin, S. Gemming, F. Wang, G. Catalan, J. F. Scott, N. A. Spaldin, J. Orenstein, and R. Ramesh, Nat. Mater. 8, 229 (2009).

${ }^{52}$ J. Seidel, D. Fu, S.-Y. Yang, E. Alarcón-Lladó, J. Wu, R. Ramesh, and J. W. Ager, Phys. Rev. Lett. 107, 126805 (2011).

${ }^{53}$ S. Y. Yang, J. Seidel, S. J. Bynes, P. Shafer, C. H. Yang, M. D. Rossell, P. Yu, Y. H. Chu, J. F. Scott, J. W. Ager III, L. W. Martin, and R. Ramesh, Nat. Nanotechnol. 5, 143 (2010).

${ }^{54}$ A. Moreira dos Santos, A. Cheetham, T. Atou, Y. Syono, Y. Yamaguchi, K. Ohoyama, H. Chiba, and C. Rao, Phys. Rev. B 66, 064425 (2002).

${ }^{55}$ T. Shishidou, N. Mikamo, Y. Uratani, F. Ishii, and T. Oguchi, J. Phys.: Condens. Matter 16, S5677 (2004).

${ }^{56}$ T. Yokosawa, A. A. Belik, T. Asaka, K. Kimoto, E. TakayamaMuromachi, and Y. Matsui, Phys. Rev. B 77, 024111 (2008).

${ }^{57}$ J. Y. Son and Y. H. Shin, Appl. Phys. Lett. 93, 062902 (2008).

${ }^{58}$ T. Kimura, S. Kawamoto, I. Yamada, M. Azuma, M. Takano, and Y. Tokura, Phys. Rev. B 67, 180401 (2003).

${ }^{59}$ E. Ohshima, Y. Saya, M. Nantoh, and M. Kawai, Solid State Commun. 116, 73 (2000).

${ }^{60}$ J. Y. Son, B. G. Kim, C. H. Kim, and J. H. Cho, Appl. Phys. Lett. 84, 4971 (2004).

${ }^{61}$ A. F. M. Santos, A. K. Cheetham, W. Tian, X. Pan, Y. Jia, N. J. Murphy, J. Lettieri, and D. G. Schlom, Appl. Phys. Lett. 84, 91 (2004).

${ }^{62}$ W. Eerenstein, F. D. Morrison, J. F. Scott, and N. D. Mathur, Appl. Phys. Lett. 87, 101906 (2005).

${ }^{63}$ C. H. Yang, T. Y. Koo, S. H. Lee, C. Song, K. B. Lee, and Y. H. Jeong, Europhys. Lett. 74, 348 (2006)

${ }^{64}$ G. M. De Luca, D. Preziosi, F. Chiarella, R. Di Capua, S. Gariglio, S. Lettieri, and M. Salluzzo, Appl. Phys. Lett. 103, 062902 (2013).

${ }^{65}$ M. Gajek, M. Bibes, F. Wyczisk, M. Varela, J. Fontcuberta, and A. Barthélémy, Phys. Rev. B 75, 174417 (2007).

${ }^{66}$ M. Gajek, M. Bibes, S. Fusil, K. Bouzehouane, J. Fontcuberta, A. Barthelemy, and A. Fert, Nat. Mater. 6, 296 (2007).

${ }^{67}$ D. H. Kim, H. N. Lee, M. Varela, and H. M. Christen, Appl. Phys. Lett. 89, 162904 (2006)

${ }^{68}$ N. A. Hill, P. Battig, and C. Daul, J. Phys. Chem. B 106, 3383 (2002).

${ }^{69}$ P. Baettig, C. Ederer, and N. Spaldin, Phys. Rev. B 72, 214105 (2005)

${ }^{70}$ A. David, P. Boullay, R. V. K. Mangalam, N. Barrier, and W. Prellier, Appl. Phys. Lett. 96, 221904 (2010).

${ }^{71}$ P. Baettig and N. A. Spaldin, Appl. Phys. Lett. 86, 012505 (2005).

${ }^{72}$ D. H. Kim, H. N. Lee, M. D. Biegalski, and H. M. Christen, Appl. Phys. Lett. 91, 042906 (2007).

${ }^{73}$ E. M. Choi, S. Patnaik, E. Weal, S. L. Sahonta, H. Wang, Z. Bi, J. Xiong, M. G. Blamire, Q. X. Jia, and J. L. Macmanus-Driscoll, Appl. Phys. Lett. 98, 012509 (2011).
${ }^{74}$ R. Nechache, P. Gupta, C. Harnagea, and A. Pignolet, Appl. Phys. Lett. 91, 222908 (2007).

${ }^{75}$ J. A. Alonso, M. J. Martinez-Lope, M. T. Casais, and M. T. FernandezDaz, Inorg. Chem. 39, 917 (2000).

${ }^{76}$ M. Nakamura, Y. Tokunaga, M. Kawasaki, and Y. Tokura, Appl. Phys. Lett. 98, 082902 (2011).

${ }^{77}$ J. H. Lee, P. Murugavel, H. Ryu, D. Lee, J. Y. Jo, J. W. Kim, H. J. Kim, K. H. Kim, Y. Jo, M. H. Jung, Y. H. Oh, Y. W. Kim, J. G. Yoon, J. S. Chung, and T. W. Noh, Adv. Mater. 18, 3125 (2006).

${ }^{78}$ B. B. Van Aken, T. T. Palstra, A. Filippetti, and N. A. Spaldin, Nat. Mater. 3, 164 (2004).

${ }^{79}$ H. L. Yakel, W. C. Koehler, E. F. Bertaut, and E. F. Forrat, Acta Crystallogr. 16, 589 (1963).

${ }^{80}$ G. A. Smolenskii and V. A. Bokov, J. Appl. Phys. 35, 915 (1964).

${ }^{81}$ D. Y. Cho, J. Y. Kim, B. G. Park, K. J. Rho, J. H. Park, H. J. Noh, B. J. Kim, S. J. Oh, H. M. Park, J. S. Ahn, H. Ishibashi, S. W. Cheong, J. Lee, P. Murugavel, T. Noh, A. Tanaka, and T. Jo, Phys. Rev. Lett. 98, 217601 (2007).

${ }^{82}$ M. Fiebig, T. Lottermoser, D. Frohlich, A. V. Goltsev, and R. V. Pisarev, Nature 419, 818 (2002).

${ }^{83}$ A. Goltsev, R. Pisarev, T. Lottermoser, and M. Fiebig, Phys. Rev. Lett. 90, 177204 (2003).

${ }^{84}$ T. Choi, Y. Horibe, H. T. Yi, Y. J. Choi, W. Wu, and S. W. Cheong, Nat. Mater. 9, 253 (2010).

${ }^{85}$ S. Lee, A. Pirogov, M. Kang, K. H. Jang, M. Yonemura, T. Kamiyama, S. W. Cheong, F. Gozzo, N. Shin, H. Kimura, Y. Noda, and J. G. Park, Nature 451, 805 (2008).

${ }^{86}$ N. Fujimura, T. Ishida, T. Yoshimura, and T. Ito, Appl. Phys. Lett. 69, 1011 (1996).

${ }^{87}$ D. Ito, N. Fujimura, T. Yoshimura, and T. Ito, J. Appl. Phys. 93, 5563 (2003).

${ }^{88}$ H. Kitahata, K. Tadanaga, T. Minami, N. Fujimura, and T. Ito, Appl. Phys. Lett. 75, 719 (1999).

${ }^{89}$ T. Yoshimura, N. Fujimura, and T. Ito, Appl. Phys. Lett. 73, 414 (1998).

${ }^{90}$ W.-C. Yi, J.-S. Choe, C.-R. Moon, S.-I. Kwun, and J.-G. Yoon, Appl. Phys. Lett. 73, 903 (1998).

${ }^{91}$ A. Posadas, J. B. Yau, C. H. Ahn, J. Han, S. Gariglio, K. Johnston, K. M. Rabe, and J. B. Neaton, Appl. Phys. Lett. 87, 171915 (2005).

${ }^{92}$ Y. Chye, T. Liu, D. Li, K. Lee, D. Lederman, and T. H. Myers, Appl. Phys. Lett. 88, 132903 (2006).

${ }^{93}$ Z. Sheng, N. Ogawa, Y. Ogimoto, and K. Miyano, Adv. Mater. 22, 5507 (2010).

${ }^{94}$ T. C. Han and H. H. Chao, Appl. Phys. Lett. 97, 232902 (2010).

${ }^{95}$ T. L. Thomas Lottermoser, U. Amann, D. Hohlwein, J. Ihringer, and M. Fiebig, Nature 430, 541 (2004).

${ }^{96}$ D. Iuşan, K. Yamauchi, P. Barone, B. Sanyal, O. Eriksson, G. Profeta, and S. Picozzi, Phys. Rev. B 87, 014403 (2013).

${ }^{97}$ S. Picozzi, K. Yamauchi, B. Sanyal, I. Sergienko, and E. Dagotto, Phys. Rev. Lett. 99, 227201 (2007).

${ }^{98}$ I. Sergienko, C. Şen, and E. Dagotto, Phys. Rev. Lett. 97, 227204 (2006).

${ }^{99}$ Y. Tokura and S. Seki, Adv. Mater. 22, 1554 (2010).

${ }^{100}$ T. Kimura, Annu. Rev. Mater. Res. 37, 387 (2007).

${ }^{101}$ I. A. Sergienko and E. Dagotto, Phys. Rev. B 73, 094434 (2006).

${ }^{102}$ T. Kimura, G. Lawes, T. Goto, Y. Tokura, and A. Ramirez, Phys. Rev. B 71, 224425 (2005)

${ }^{103}$ C. J. Daumont, D. Mannix, S. Venkatesan, G. Catalan, D. Rubi, B. J. Kooi, J. T. De Hosson, and B. Noheda, J. Phys.: Condens. Matter 21, 182001 (2009).

${ }^{104}$ D. Rubi, C. de Graaf, C. Daumont, D. Mannix, R. Broer, and B. Noheda, Phys. Rev. B 79, 014416 (2009).

${ }^{105}$ S. Venkatesan, C. Daumont, B. J. Kooi, B. Noheda, and J. T. M. D. Hosson, Phys. Rev. B 80, 214111 (2009).

${ }^{106}$ B. J. Kirby, D. Kan, A. Luykx, M. Murakami, D. Kundaliya, and I. Takeuchi, J. Appl. Phys. 105, 07D917 (2009).

${ }^{107}$ X. Marti, V. Skumryev, C. Ferrater, M. V. García-Cuenca, M. Varela, F. Sánchez, and J. Fontcuberta, Appl. Phys. Lett. 96, 222505 (2010).

${ }^{108}$ X. Marti, V. Skumryev, A. Cattoni, R. Bertacco, V. Laukhin, C. Ferrater, M. V. García-Cuenca, M. Varela, F. Sánchez, and J. Fontcuberta, J. Magn. Magn. Mater. 321, 1719 (2009).

${ }^{109}$ N. Jehanathan, O. Lebedev, I. Gelard, C. Dubourdieu, and G. Van Tendeloo, Nanotechnology 21, 75705 (2010).

${ }^{110}$ A. Glavic, C. Becher, J. Voigt, E. Schierle, E. Weschke, M. Fiebig, and T. Brückel, Phys. Rev. B 88, 054401 (2013). 
${ }^{111}$ C. L. Lu, S. Dong, Z. C. Xia, H. Luo, Z. B. Yan, H. W. Wang, Z. M. Tian, S. L. Yuan, T. Wu, and J.-M. Liu, Sci. Rep. 3, 3374 (2013).

${ }^{112}$ X. Li, C. L. Lu, J. Y. Dai, S. Dong, Y. Chen, N. Hu, G. H. Wu, M. F. Liu, Z. B. Yan, and J.-M. Liu, Sci. Rep. 4, 7019 (2014).

${ }^{113}$ I. Fina, L. Fàbrega, X. Martí, F. Sánchez, and J. Fontcuberta, Phys. Rev. Lett. 107, 257601 (2011).

${ }^{114}$ F. Jiménez-Villacorta, J. A. Gallastegui, I. Fina, X. Marti, and J. Fontcuberta, Phys. Rev. B 86, 024420 (2012).

${ }^{115}$ R. D. Johnson, L. C. Chapon, D. D. Khalyavin, P. Manuel, P. G. Radaelli, and C. Martin, Phys. Rev. Lett. 108, 067201 (2012).

${ }^{116}$ V. Caignaert, A. Maignan, K. Singh, C. Simon, V. Pralong, B. Raveau, J. F. Mitchell, H. Zheng, A. Huq, and L. C. Chapon, Phys. Rev. B 88, $174403(2013)$

${ }^{117}$ T. Aoyama, K. Yamauchi, A. Iyama, S. Picozzi, K. Shimizu, and T. Kimura, Nat. Commun. 5, 4927 (2014).

${ }^{118}$ S. Seki, Y. Onose, and Y. Tokura, Phys. Rev. Lett. 101, 067204 (2008).

${ }^{119}$ S. Luo, K. F. Wang, S. Z. Li, X. W. Dong, Z. B. Yan, H. L. Cai, and J. M. Liu, Appl. Phys. Lett. 94, 172504 (2009).

${ }^{120}$ K. Kimura, H. Nakamura, S. Kimura, M. Hagiwara, and T. Kimura, Phys. Rev. Lett. 103, 107201 (2009).

${ }^{121}$ H. Hiraga, T. Fukumura, A. Ohtomo, T. Makino, A. Ohkubo, H. Kimura, and M. Kawasaki, Appl. Phys. Lett. 95, 032109 (2009).

${ }^{122}$ H. Hiraga, T. Makino, T. Fukumura, H. Weng, and M. Kawasaki, Phys. Rev. B 84, 041411 (2011).

${ }^{123}$ M. B. Holcomb, L. W. Martin, A. Scholl, Q. He, P. Yu, C. H. Yang, S. Y. Yang, P. A. Glans, M. Valvidares, M. Huijben, J. B. Kortright, J. Guo, Y. H. Chu, and R. Ramesh, Phys. Rev. B 81, 134406 (2010).

${ }^{124}$ C.-J. Cheng, C. Lu, Z. Chen, L. You, L. Chen, J. Wang, and T. Wu, Appl. Phys. Lett. 98, 242502 (2011)

${ }^{125}$ M. Singh, W. Prellier, M. Singh, R. Katiyar, and J. Scott, Phys. Rev. B 77, 144403 (2008)

${ }^{126}$ V. A. Khomchenko, D. A. Kiselev, J. M. Vieira, L. Jian, A. L. Kholkin, A. M. L. Lopes, Y. G. Pogorelov, J. P. Araujo, and M. Maglione, J. Appl. Phys. 103, 024105 (2008).

${ }^{127}$ S. Zhang, W. Luo, D. Wang, and Y. Ma, Mater. Lett. 63, 1820 (2009).

${ }^{128}$ G. L. Yuan, S. W. Or, J. M. Liu, and Z. G. Liu, Appl. Phys. Lett. 89, 052905 (2006)

${ }^{129}$ T. J. Park, G. C. Papaefthymiou, A. J. Viescas, A. R. Moodenbaugh, and S. S. Wong, Nano Lett. 7, 766 (2007).

${ }^{130}$ F. Gao, Y. Yuan, K. F. Wang, X. Y. Chen, F. Chen, J. M. Liu, and Z. F. Ren, Appl. Phys. Lett. 89, 102506 (2006).

${ }^{131}$ X. Marti, V. Skumryev, V. Laukhin, R. Bachelet, C. Ferrater, M. V. García-Cuenca, M. Varela, F. Sánchez, and J. Fontcuberta, J. Appl. Phys. 108, 123917 (2010).

${ }^{132}$ J. S. White, M. Bator, Y. Hu, H. Luetkens, J. Stahn, S. Capelli, S. Das, M. Döbeli, T. Lippert, V. K. Malik, J. Martynczuk, A. Wokaun, M. Kenzelmann, C. Niedermayer, and C. W. Schneider, Phys. Rev. Lett. 111, 037201 (2013).

${ }^{133}$ C. Wang, K.-j. Jin, Z.-t. Xu, L. Wang, C. Ge, H.-b. Lu, H.-z. Guo, M. He, and G.-z. Yang, Appl. Phys. Lett. 98, 192901 (2011).

${ }^{134}$ A. Q. Jiang, C. Wang, K. J. Jin, X. B. Liu, J. F. Scott, C. S. Hwang, T. A. Tang, H. B. Lu, and G. Z. Yang, Adv. Mater. 23, 1277 (2011).

${ }^{135}$ T. Choi, S. Lee, Y. J. Choi, V. Kiryukhin, and S. W. Cheong, Science 324, 63 (2009).

${ }^{136}$ A. Tsurumaki, H. Yamada, and A. Sawa, Adv. Funct. Mater. 22, 1040 (2012).

${ }^{137}$ Z. B. Yan, S. Z. Li, K. F. Wang, and J. M. Liu, Appl. Phys. Lett. 96, 012103 (2010)

${ }^{138}$ Z. B. Yan and J. M. Liu, Sci. Rep. 3, 2482 (2013).

${ }^{139}$ Y. Cui, H. Peng, S. Wu, R. Wang, and T. Wu, ACS Appl. Mater. Interfaces 5, 1213 (2013).

${ }^{140}$ E. Y. Tsymbal and H. Kohlstedt, Science 313, 181 (2006).

${ }^{141}$ D. D. Fong, G. B. Stephenson, S. K. Streiffer, J. A. Eastman, O. Auciello, P. H. Fuoss, and C. Thompson, Science 304, 1650 (2004).

${ }^{142}$ A. Chanthbouala, V. Garcia, R. O. Cherifi, K. Bouzehouane, S. Fusil, X. Moya, S. Xavier, H. Yamada, C. Deranlot, N. D. Mathur, M. Bibes, A. Barthelemy, and J. Grollier, Nat. Mater. 11, 860 (2012).

${ }^{143}$ H. Yamada, V. Garcia, S. Fusil, S. Boyn, M. Marinova, A. Gloter, S. Xavier, J. Grollier, E. Jacquet, C. Carretero, C. Deranlot, M. Bibes, and A. Barthelemy, ACS Nano 7, 5385 (2013).

${ }^{144}$ D. Pantel, S. Goetze, D. Hesse, and M. Alexe, Nat. Mater. 11, 289 (2012).

${ }^{145}$ E. Y. Tsymbal and A. Gruverman, Nat. Mater. 12, 602 (2013).
${ }^{146}$ Z. Wen, C. Li, D. Wu, A. D. Li, and N. B. Ming, Nat. Mater. 12, 617 (2013).

${ }^{147}$ D. J. Kim, T. R. Paudel, H. Lu, J. D. Burton, J. G. Connell, E. Y. Tsymbal, S. S. Ambrose Seo, and A. Gruverman, Adv. Mater. 26, 7660 (2014).

${ }^{148}$ C. A. Fernandes Vaz and U. Staub, J. Mater. Chem. C 1, 6731 (2013).

${ }^{149}$ L. You, C. Lu, P. Yang, G. Han, T. Wu, U. Luders, W. Prellier, K. Yao, L. Chen, and J. Wang, Adv. Mater. 22, 4964 (2010).

${ }^{150}$ L. W. Martin, Y. H. Chu, M. B. Holcomb, M. Huijben, P. Yu, S. J. Han, D. Lee, S. X. Wang, and R. Ramesh, Nano Lett. 8, 2050 (2008).

${ }^{151}$ L. W. Martin, Y.-H. Chu, Q. Zhan, R. Ramesh, S.-J. Han, S. X. Wang, M. Warusawithana, and D. G. Schlom, Appl. Phys. Lett. 91, 172513 (2007).

${ }^{152}$ Y. H. Chu, L. W. Martin, M. B. Holcomb, M. Gajek, S. J. Han, Q. He, N. Balke, C. H. Yang, D. Lee, W. Hu, Q. Zhan, P. L. Yang, A. FraileRodriguez, A. Scholl, S. X. Wang, and R. Ramesh, Nat. Mater. 7, 478 (2008).

${ }^{153}$ J. Allibe, I. C. Infante, S. Fusil, K. Bouzehouane, E. Jacquet, C. Deranlot, M. Bibes, and A. Barthélémy, Appl. Phys. Lett. 95, 182503 (2009).

${ }^{154}$ J. Dho and M. G. Blamire, J. Appl. Phys. 106, 073914 (2009).

${ }^{155}$ H. Naganuma, M. Oogane, and Y. Ando, J. Appl. Phys. 109, $07 D 736$ (2011).

${ }^{156}$ S. M. Wu, S. A. Cybart, D. Yi, J. M. Parker, R. Ramesh, and R. C. Dynes, Phys. Rev. Lett. 110, 067202 (2013).

${ }^{157}$ S. M. Wu, S. A. Cybart, P. Yu, M. D. Rossell, J. X. Zhang, R. Ramesh, and R. C. Dynes, Nat. Mater. 9, 756 (2010).

${ }^{158}$ P. Yu, J. S. Lee, S. Okamoto, M. D. Rossell, M. Huijben, C. H. Yang, Q. He, J. X. Zhang, S. Y. Yang, M. J. Lee, Q. M. Ramasse, R. Erni, Y. H. Chu, D. A. Arena, C. C. Kao, L. W. Martin, and R. Ramesh, Phys. Rev. Lett. 105, 027201 (2010).

${ }^{159}$ H. Béa, M. Bibes, S. Cherifi, F. Nolting, B. Warot-Fonrose, S. Fusil, G. Herranz, C. Deranlot, E. Jacquet, K. Bouzehouane, and A. Barthélémy, Appl. Phys. Lett. 89, 242114 (2006).

${ }^{160}$ Y. F. Tian, J. F. Ding, W. N. Lin, Z. H. Chen, A. David, M. He, W. J. Hu, L. Chen, and T. Wu, Sci. Rep. 3, 1094 (2013).

${ }^{161}$ V. Laukhin, V. Skumryev, X. Martí, D. Hrabovsky, F. Sánchez, M. García-Cuenca, C. Ferrater, M. Varela, U. Lüders, J. Bobo, and J. Fontcuberta, Phys. Rev. Lett. 97, 227201 (2006).

${ }^{162}$ L. You, B. Wang, X. Zou, Z. S. Lim, Y. Zhou, H. Ding, L. Chen, and J. Wang, Phys. Rev. B 88, 184426 (2013).

${ }^{163}$ J. T. Heron, J. L. Bosse, Q. He, Y. Gao, M. Trassin, L. Ye, J. D. Clarkson, C. Wang, J. Liu, S. Salahuddin, D. C. Ralph, D. G. Schlom, J. Iniguez, B. D. Huey, and R. Ramesh, Nature 516, 370 (2014).

${ }^{164}$ T. Zhao, A. Scholl, F. Zavaliche, K. Lee, M. Barry, A. Doran, M. P. Cruz, Y. H. Chu, C. Ederer, N. A. Spaldin, R. R. Das, D. M. Kim, S. H. Baek, C. B. Eom, and R. Ramesh, Nat. Mater. 5, 823 (2006).

${ }^{165}$ J. Allibe, S. Fusil, K. Bouzehouane, C. Daumont, D. Sando, E. Jacquet, C. Deranlot, M. Bibes, and A. Barthelemy, Nano Lett. 12, 1141 (2012).

${ }^{166}$ Y.-M. Kim, A. Morozovska, E. Eliseev, M. P. Oxley, R. Mishra, S. M. Selbach, T. Grande, S. T. Pantelides, S. V. Kalinin, and A. Y. Borisevich, Nat. Mater. 13, 1019 (2014).

${ }^{167}$ M. Bibes and A. Barthélémy, Nat. Mater. 7, 425 (2008).

${ }^{168}$ H. Béa, M. Bibes, F. Ott, B. Dupé, X. H. Zhu, S. Petit, S. Fusil, C. Deranlot, K. Bouzehouane, and A. Barthélémy, Phys. Rev. Lett. 100, 017204 (2008).

${ }^{169}$ K. L. Livesey, Phys. Rev. B 82, 064408 (2010).

${ }^{170}$ S. Dong, K. Yamauchi, S. Yunoki, R. Yu, S. Liang, A. Moreo, J. M. Liu, S. Picozzi, and E. Dagotto, Phys. Rev. Lett. 103, 127201 (2009).

${ }^{171}$ W. Ramadan, S. Ogale, S. Dhar, L. Fu, S. Shinde, D. Kundaliya, M. Rao, N. Browning, and T. Venkatesan, Phys. Rev. B 72, 205333 (2005).

${ }^{172}$ J. R. Sun, C. M. Xiong, Y. Z. Zhang, and B. G. Shen, Appl. Phys. Lett. 87, 222501 (2005)

${ }^{173}$ R. Ramesh, A. Inam, W. K. Chan, F. Tillerot, B. Wilkens, C. C. Chang, T. Sands, J. M. Tarascon, and V. G. Keramidas, Appl. Phys. Lett. 59, 3542 (1991).

${ }^{174}$ H. J. Zhang, X. P. Zhang, J. P. Shi, H. F. Tian, and Y. G. Zhao, Appl. Phys. Lett. 94, 092111 (2009).

${ }^{175}$ C. H. Ahn, J. M. Triscone, and J. Mannhart, Nature 424, 1015 (2003).

${ }^{176}$ C. L. Lu, Y. Wang, L. You, X. Zhou, H. Y. Peng, G. Z. Xing, E. E. M. Chia, C. Panagopoulos, L. Chen, J.-M. Liu, J. Wang, and T. Wu, Appl. Phys. Lett. 97, 252905 (2010).

${ }^{177}$ A. Crassous, R. Bernard, S. Fusil, K. Bouzehouane, D. Le Bourdais, S. E. Vedrenne, J. Briatico, M. Bibes, A. Barthélémy, and J. E. Villegas, Phys. Rev. Lett. 107, 247002 (2011).

${ }^{178}$ W. Ji, K. Yao, and Y. C. Liang, Adv. Mater. 22, 1763 (2010).

${ }^{179}$ W. Ji, K. Yao, and Y. C. Liang, Phys. Rev. B 84, 094115 (2011). 
${ }^{180}$ S. R. Basu, L. W. Martin, Y. H. Chu, M. Gajek, R. Ramesh, R. C. Rai, X. Xu, and J. L. Musfeldt, Appl. Phys. Lett. 92, 091905 (2008).

${ }^{181}$ M. Qin, K. Yao, and Y. C. Liang, Appl. Phys. Lett. 95, 022912 (2009).

${ }^{182}$ M. Qin, K. Yao, Y. C. Liang, and B. K. Gan, Appl. Phys. Lett. 91, 092904 (2007)

${ }^{183}$ K. Yao, B. K. Gan, M. Chen, and S. Shannigrahi, Appl. Phys. Lett. 87, 212906 (2005).

${ }^{184}$ A. Bhatnagar, A. Roy Chaudhuri, Y. Heon Kim, D. Hesse, and M. Alexe, Nat. Commun. 4, 2835 (2013)
${ }^{185}$ A. Bhatnagar, Y. H. Kim, D. Hesse, and M. Alexe, Nano Lett. 14, 5224 (2014).

${ }^{186}$ G.-L. Yuan and J. Wang, Appl. Phys. Lett. 95, 252904 (2009).

${ }^{187}$ H. T. Yi, T. Choi, S. G. Choi, Y. S. Oh, and S. W. Cheong, Adv. Mater. 23, 3403 (2011).

${ }^{188}$ R. Guo, L. You, Y. Zhou, Z. S. Lim, X. Zou, L. Chen, R. Ramesh, and J. L. Wang, Nat. Commun. 4, 1990 (2013).

${ }^{189}$ B. Kundys, M. Viret, D. Colson, and D. O. Kundys, Nat. Mater. 9, 803 (2010). 